\title{
Vacuum energy in the effective field theory of general relativity
}

\author{
J. Gegelia ${ }^{1,2}$ and Ulf-G. Meißner, ${ }^{3,4,2}$ \\ ${ }^{1}$ Ruhr University Bochum, Faculty of Physics and Astronomy, Institute for Theoretical Physics II, \\ D-44870 Bochum, Germany \\ ${ }^{2}$ Tbilisi State University, 0186 Tbilisi, Georgia \\ ${ }^{3}$ Helmholtz Institut für Strahlen- und Kernphysik and Bethe Center for Theoretical Physics, \\ Universität Bonn, D-53115 Bonn, Germany \\ ${ }^{4}$ Institute for Advanced Simulation, Institut für Kernphysik and Jülich Center for Hadron Physics, \\ Forschungszentrum Jülich, D-52425 Jülich, Germany
}

(Received 3 June 2019; published 28 August 2019)

\begin{abstract}
In the framework of an effective field theory of general relativity, a model of scalar and vector bosons interacting with the metric field is considered. It is shown in the framework of a two-loop order calculation that, for the cosmological constant term which is fixed by the condition of vanishing vacuum energy, the graviton remains massless and there exists a self-consistent effective field theory of general relativity coupled to matter fields defined on a flat Minkowski background. This result is obtained under the assumption that the energy-momentum tensor of the gravitational field is given by the pseudotensor of Landau-Lifshitz's classic textbook. Implications for the cosmological constant problem are also briefly discussed.
\end{abstract}

DOI: 10.1103/PhysRevD.100.046021

\section{INTRODUCTION}

It is widely accepted that, whatever the underlying fundamental theory of all interactions might be, at low energies the physics can be adequately described by an effective field theory (EFT) [1]. Gravitation can also be included in the formalism of the EFT by considering the most general effective Lagrangian of metric fields interacting with matter fields $[2,3]$ which is invariant under all underlying symmetries, including the gauge symmetry of massless spin-two particles [4]. This quantum field theoretical treatment of general relativity with the metric field presented as the Minkowski background plus the graviton field and the cosmological constant usually set equal to zero is considered as a well-defined approach in the modern sense; see, e.g., Ref. [5]. It is well known that, for a nonvanishing cosmological constant term $\Lambda$, the graviton propagator has a pole corresponding to a massive ghost mode [4]. Setting $\Lambda$ equal to zero, as is usually done in the EFT of gravitation [2], does not solve the problem, as the radiative corrections regenerate the problem with the massive ghost [6]. This is because the cosmological constant term is not suppressed by any symmetry of the

Published by the American Physical Society under the terms of the Creative Commons Attribution 4.0 International license. Further distribution of this work must maintain attribution to the author(s) and the published article's title, journal citation, and DOI. Funded by SCOAP ${ }^{3}$. effective theory, and therefore there is no protection against generating such a contribution to the effective action by radiative corrections. However, as has been shown in Ref. [6], one can represent the cosmological constant as a power series in $\hbar$ and choose the coefficients of this series such that the graviton becomes a massless spin-2 particle up to all orders in the loop expansion. Thus, within a perturbative EFT in a flat Minkowski background, the cosmological constant, which is one of the parameters of the effective Lagrangian, is uniquely fixed. This does not solve the cosmological constant problem [7] (for a recent review of the cosmological constant problem, see, e.g., Ref. [8]) but rather implies that taking into account a cosmological constant term other than that obtained in Ref. [6] necessarily requires considering an EFT in a curved background field. In this case, by imposing the equations of motion with respect to the background graviton field, the mass term of the graviton is removed at tree level [9]; however, a systematic study of the issue at higher orders in loop expansion requires an EFT on a curved background metric which, to the best of our knowledge, is not available yet.

Experimental evidence of the accelerating expansion of the Universe (see, e.g., Ref. [10] and the references therein) leaves us with a very challenging problem-namely, the huge discrepancy between the measured small value of the cosmological constant and its theoretical estimation [7]. An important question related to this problem is whether there exists any condition that uniquely fixes the value of the 
cosmological constant. It seems natural to expect the energy of the physical vacuum state of the theory describing the Universe to be exactly zero. That is the main assumption of this work, and it is of importance for the later discussions. In the framework of the low-energy EFT of general relativity coupled to the fields of the Standard Model, imposing such a condition uniquely fixes the cosmological constant term as a function of other parameters of the effective Lagrangian. In this work we calculate the vacuum expectation value of the full four-momentum of the gravitational and matter fields at two-loop order in a simplified version of the Abelian model, with spontaneous symmetry breaking considered in Ref. [6]. We obtain that, as a result of a nontrivial cancellation between different diagrams, the vacuum energy exactly vanishes for the value of the cosmological constant obtained in Ref. [6], i.e., for the value which guarantees the vanishing of the graviton mass and the vacuum expectation value of the graviton field at two-loop order. That is, provided that our result holds to all orders, the uniquely fixed value of the cosmological constant term, leading to a self-consistent perturbative EFT on the Minkowki background is obtained as a consequence of imposing the condition of vanishing vacuum energy. Notice here that, being aware of the lack of a commonly accepted expression of the energy-momentum tensor for the gravitational field (see, e.g., Refs. [11-15]), in this work we use the definition of the energy-momentum pseudotensor and the full four-momentum of the matter and gravitational fields given in the classic textbook by Landau and Lifshitz [17].

Our work is organized as follows: In Sec. II we specify the details of the considered EFT and calculate one- and two-loop order contributions to the vacuum energy. In Sec. III we briefly discuss the implications of the obtained results on the cosmological constant problem. We summarize in Sec. IV, and the Appendix contains the Feynman rules and two-loop integrals required for our calculations.

\section{VACUUM ENERGY IN AN EFT OF GENERAL RELATIVITY ON A MINKOWSKI BACKGROUND}

In the framework of EFT the action of matter interacting with gravity is given by the most general effective Lagrangian of gravitational and matter fields, which is invariant under general coordinate transformations and other symmetries of the Standard Model,

$$
\begin{aligned}
S & =\int d^{4} x \sqrt{-g}\left\{\mathcal{L}_{\mathrm{gr}}(g)+\mathcal{L}_{\mathrm{m}}(g, \psi)\right\} \\
& =\int d^{4} x \sqrt{-g}\left\{\frac{2}{\kappa^{2}}(R-2 \Lambda)+\mathcal{L}_{\mathrm{gr}, \mathrm{ho}}(g)+\mathcal{L}_{\mathrm{m}}(g, \psi)\right\} \\
& =S_{\mathrm{gr}}(g)+S_{\mathrm{m}}(g, \psi),
\end{aligned}
$$

where $\kappa^{2}=32 \pi G$, with $G=6.70881 \times 10^{-39} \mathrm{GeV}^{-2}$ being the gravitational (Newton's) constant, $\psi$ and $g^{\mu \nu}$ denote the matter and metric fields, respectively, $g=\operatorname{det} g^{\mu \nu}, \Lambda$ is the cosmological constant and $R$ is the scalar curvature. Further, $\mathcal{L}_{\text {gr,ho }}(g)$ represents selfinteraction terms of the gravitational field with higher orders of derivatives, and $\mathcal{L}_{\text {matter }}(g, \psi)$ is the effective Lagrangian of the matter fields interacting with gravity. Experimental evidence suggests that the contributions of $\mathcal{L}_{\text {gr,ho }}(g)$, as well as the contributions of nonrenormalizable interactions of $\mathcal{L}_{\text {matter }}(g, \psi)$ in physical quantities, are heavily suppressed. Vielbein tetrad fields have to be introduced for fermionic fields interacting with the gravitational field; however, we refrain from giving details on these, as later we will perform calculations with bosonic degrees of freedom only.

The low-energy EFT of general relativity is obtained by representing the gravitational field as the sum of the Minkowskian background and the quantum fields [16]

$$
\begin{aligned}
g_{\mu \nu} & =\eta_{\mu \nu}+\kappa h_{\mu \nu}, \\
g^{\mu \nu} & =\eta^{\mu \nu}-\kappa h^{\mu \nu}+\kappa^{2} h_{\lambda}^{\mu} h^{\lambda \nu}-\kappa^{3} h_{\lambda}^{\mu} h_{\sigma}^{\lambda} h^{\sigma \nu}+\cdots,
\end{aligned}
$$

and by calculating physical quantities perturbatively by applying the standard quantum field theory technique.

The energy-momentum tensor of the matter fields coupled to the gravitational field, $T_{\mathrm{m}}^{\mu \nu}$, and the pseudotensor of the gravitational field, $T_{L L}^{\mu \nu}$, are given by

$$
\begin{gathered}
T_{\mathrm{m}}^{\mu \nu}(g, \psi)=\frac{2}{\sqrt{-g}} \frac{\delta S_{\mathrm{m}}}{\delta g_{\mu \nu}}, \\
T_{\mathrm{gr}}^{\mu \nu}(g)=\frac{4}{\kappa^{2}} \Lambda g^{\mu \nu}+T_{L L}^{\mu \nu}(g),
\end{gathered}
$$

where $T_{L L}^{\mu \nu}(g)$ is defined via [17]

$$
\begin{aligned}
& (-g) T_{L L}^{\mu \nu}(g)=\frac{2}{\kappa^{2}}\left(\frac{1}{8} g^{\lambda \sigma} g^{\mu \nu} g_{\alpha \gamma} g_{\beta \delta} \mathfrak{g}^{\alpha \gamma}{ }_{\sigma} \mathfrak{g}^{\beta \delta}{ }_{\lambda}-\frac{1}{4} g^{\mu \lambda} g^{\nu \sigma} g_{\alpha, \gamma} g_{\beta \delta} \mathfrak{g}^{\alpha \gamma}{ }_{\sigma} \mathfrak{g}^{\beta \delta},{ }_{\lambda}-\frac{1}{4} g^{\lambda \sigma} g^{\mu \nu} g_{\beta \alpha} g_{\gamma \delta} \mathfrak{g}^{\alpha \gamma}{ }_{\sigma} \mathfrak{g}^{\beta \delta},{ }_{\lambda}\right. \\
& +\frac{1}{2} g^{\mu \lambda} g^{\nu \sigma} g_{\beta \alpha} g_{\gamma \delta} \mathfrak{g}^{\alpha \gamma}{ }_{\sigma} \mathfrak{g}^{\beta \delta}{ }_{\lambda}+g^{\beta \alpha} g_{\lambda \sigma} \mathfrak{g}^{\nu \sigma},_{\alpha} \mathfrak{g}^{\mu \lambda}{ }_{, \beta}+\frac{1}{2} g^{\mu \nu} g_{\lambda \sigma} \mathfrak{g}^{\lambda \beta}{ }_{, \alpha} \mathfrak{g}^{\alpha \sigma}{ }_{, \beta} \\
& \left.-g^{\mu \lambda} g_{\sigma \beta} \mathfrak{g}^{\nu \beta}{ }_{\alpha} \mathfrak{g}^{\sigma \alpha},{ }_{\lambda}-g^{\nu \lambda} g_{\sigma \beta} \mathfrak{g}^{\mu \beta},_{\alpha} \mathfrak{g}^{\sigma \alpha}{ }_{\lambda}+\mathfrak{g}^{\lambda \sigma}{ }_{\sigma} \mathfrak{g}^{\mu \nu},{ }_{\lambda}-\mathfrak{g}^{\mu \lambda}, \lambda \mathfrak{g}^{\nu \sigma}{ }_{\sigma}\right),
\end{aligned}
$$


with $\mathfrak{g}^{\mu \nu}=\sqrt{-g} g^{\mu \nu}$ and $\mathfrak{g}^{\mu \nu}{ }_{, \lambda}=\partial \mathfrak{g}^{\mu \nu} / \partial x^{\lambda}$.

The full energy-momentum tensor $T^{\mu \nu}=T_{\mathrm{m}}^{\mu \nu}(g, \psi)+$ $T_{\mathrm{gr}}^{\mu \nu}(g)$ defines the conserved full four-momentum of the matter and the gravitational field as [17]

$$
P^{\mu}=\int(-g) T^{\mu \nu} d S_{\nu}
$$

where the integration is carried out over any hypersurface containing the whole three-dimensional space. Thus, the energy of the vacuum will be zero if the vacuum expectation value of the energy-momentum tensor times $(-g)$ vanishes. This quantity is given by the following path integral:

$$
\begin{aligned}
\left\langle 0\left|(-g) T^{\mu \nu}\right| 0\right\rangle= & \int \mathcal{D} g \mathcal{D} \psi(-g)\left[T_{\mathrm{gr}}^{\mu \nu}(g)+T_{\mathrm{m}}^{\mu \nu}(g, \psi)\right] \\
& \times \exp \left\{i \int d^{4} x \sqrt{-g}\left[\mathcal{L}(g, \psi)+\mathcal{L}_{\mathrm{GF}}\right]\right\},
\end{aligned}
$$

where $\mathcal{L}_{\mathrm{GF}}$ is the gauge fixing term, and the Faddeev-Popov determinant is included in the integration measure. The cosmological constant $\Lambda$ can be uniquely fixed by demanding that the right-hand side of Eq. (7) vanishes. To demonstrate how one obtains a self-consistent EFT by imposing this condition, we consider a simple model of a massive scalar and a massive vector field interacting with a metric tensor field. It coincides with the bosonic part of the model with spontaneously broken Abelian gauge symmetry considered in Ref. [6] taken in unitary gauge for the Abelian gauge symmetry. The action of the matter part of the model is given by

$$
\begin{aligned}
S_{\mathrm{m}}= & \int d^{4} x \sqrt{-g}\left\{-\frac{1}{4} g^{\mu \rho} g^{\nu \sigma} F_{\mu \nu} F_{\rho \sigma}+\frac{M^{2}}{2} g^{\mu \nu} A_{\mu} A_{\nu}\right. \\
& \left.+\frac{g^{\mu \nu}}{2} \partial_{\mu} H \partial_{\nu} H-\frac{m^{2}}{2} H^{2}+\mathcal{L}_{\mathrm{MI}}\right\},
\end{aligned}
$$

where $F_{\mu \nu}=\partial_{\mu} A_{\nu}-\partial_{\nu} A_{\mu}, A_{\mu}$ is the vector field, $H$ is the scalar field, and $\mathcal{L}_{\mathrm{MI}}$ denotes the interactions of matter fields, the specific form of which is not important for this work, as we will not include them in our calculations. The energy-momentum tensor corresponding to Eq. (8) has the form

$$
\begin{aligned}
T_{m}^{\mu \nu}= & -g^{\mu \alpha} g^{\nu \rho} g^{\beta \sigma} F_{\alpha \beta} F_{\rho \sigma}+M^{2} g^{\mu \alpha} g^{\nu \beta} A_{\alpha} A_{\beta}+\partial_{\mu} H \partial_{\nu} H \\
& -g^{\mu \nu}\left\{-\frac{1}{4} g^{\alpha \rho} g^{\beta \sigma} F_{\alpha \beta} F_{\rho \sigma}+\frac{M^{2}}{2} g^{\alpha \beta} A_{\alpha} A_{\beta}\right. \\
& \left.+\frac{g^{\alpha \beta}}{2} \partial_{\alpha} H \partial_{\beta} H-\frac{m^{2}}{2} H^{2}\right\}+T_{\mathrm{MI}}^{\mu \nu},
\end{aligned}
$$

where $T_{\mathrm{MI}}^{\mu \nu}$ corresponds to $\mathcal{L}_{\mathrm{MI}}$.
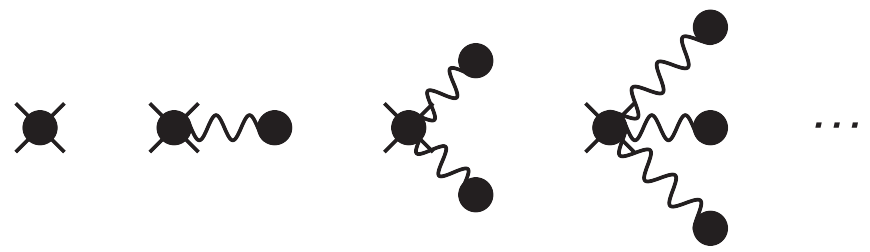

FIG. 1. Diagrams contributing to the vacuum expectation value of the energy-momentum pseudotensor times $(-g)$ at tree order. Filled circles correspond to the cosmological constant term. The cross stands for the energy-momentum pseudotensor times $(-g)$, and the wiggly line represents the graviton.

By adding the following gauge fixing term to the effective Lagrangian

$$
\mathcal{L}_{\mathrm{GF}}=\xi\left(\partial_{\nu} h^{\mu \nu}-\frac{1}{2} \partial^{\mu} h_{\nu}^{\nu}\right)\left(\partial^{\beta} h_{\mu \beta}-\frac{1}{2} \partial_{\mu} h_{\alpha}^{\alpha}\right),
$$

where $\xi$ is the gauge parameter, we obtain the Feynman rules specified in the Appendix.

For the vacuum expectation value of the full energymomentum pseudotensor times $(-g)$ at tree order, we obtain an infinite number of diagrams shown in Fig. 1. All of these contributions vanish if we take the cosmological constant vanishing at tree order. That is, we represent $\Lambda$ as

$$
\Lambda=\sum_{i=0}^{\infty} \hbar^{i} \Lambda_{i}
$$

and take $\Lambda_{0}=0$. Notice that this also removes the graviton mass from the propagator at tree order.

Next, using the Feynman rules given in the Appendix, we calculated the one-loop contributions to the vacuum expectation value of the full energy-momentum pseudotensor times $(-g)$ shown in Fig. 2, and, by demanding that $\Lambda_{1}$ cancels this contribution, we obtain (in the calculations of the loop diagrams below, we used the program FeynCalc $[18,19])$

$$
\Lambda_{1}=-\frac{\kappa^{2} \Gamma\left(1-\frac{d}{2}\right)\left(m^{d}+(d-1) M^{d}\right)}{2^{d+6} \pi^{\frac{d}{2}+4} d} .
$$

It is a trivial consequence of Eq. (3) that the same value of $\Lambda_{1}$ cancels the one-loop contribution to the vacuum expectation value of the graviton field $h_{\mu \nu}$, shown in Fig. 3, and consequently the graviton self-energy at zero momentum, i.e., the graviton mass, as a result of a Ward identity [6]. The first nontrivial result is obtained at twoloop order by calculating the diagrams contributing to the vacuum expectation value of the full energy-momentum pseudotensor times $(-g)$ shown in Fig. 2. We also calculated the two-loop contributions to the vacuum 

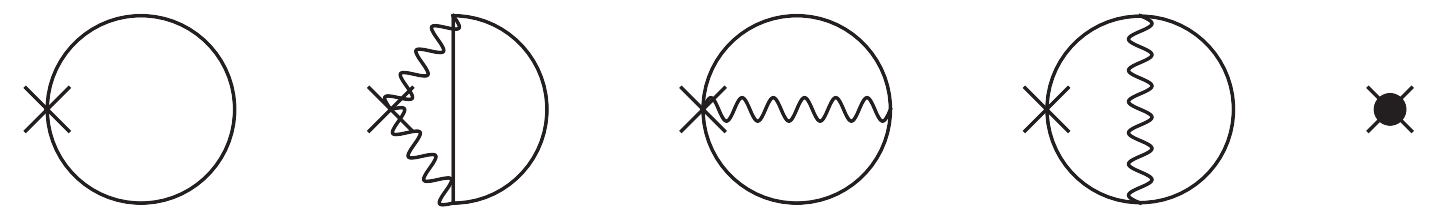

FIG. 2. Diagrams contributing to the vacuum expectation value of the energy-momentum pseudotensor times $(-g)$. The filled circle corresponds to the cosmological constant term. The cross stands for the energy-momentum pseudotensor times $(-g)$, and the wiggly and solid lines represent the graviton and the scalar (vector), respectively.
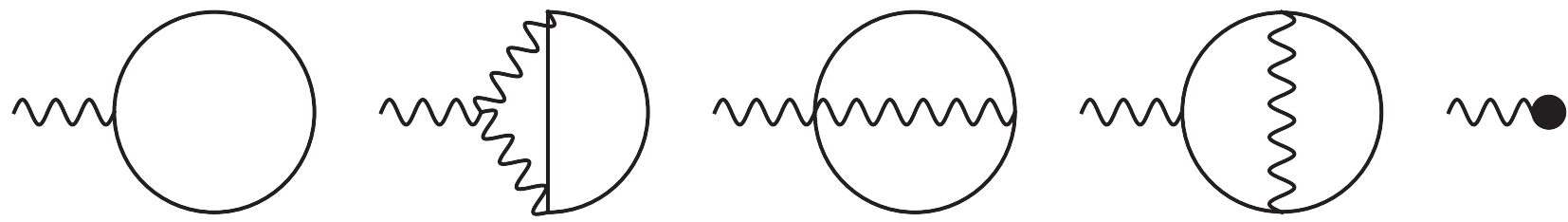

FIG. 3. Diagrams contributing to the graviton tadpole. The filled circle corresponds to the cosmological constant term. Wiggly and solid lines represent the graviton and scalar (vector) fields, respectively.

expectation value of the gravitational field shown in Fig. 3 and verified that the same value of $\Lambda_{2}$ cancels both quantities. The obtained result reads

$$
\Lambda_{2}=-\frac{d(d+1) \kappa^{4} M^{2 d-2} \csc \left(\frac{\pi d}{2}\right) \Gamma\left(1-\frac{d}{2}\right)}{2^{2(d+3)} \pi^{d-1} \Gamma\left(\frac{d}{2}\right)} .
$$

While it is a trivial consequence of Eq. (3) that the fourth diagrams in Figs. 2 and 3 both give equal contributions in $\Lambda$, it is only the sum of the corresponding second and third diagrams that lead to identical expressions. To check the obtained results, we also calculated two-loop contributions to the graviton self-energy at zero momentum and verified that the same value of $\Lambda_{2}$ cancels the twoloop order contribution to the graviton mass in agreement with the Ward identity [6] (we do not give the expressions of the corresponding Feynman rules due to their huge size). While we expect an analogous result to hold to all orders, we are not able to give a general argument supporting it.

We briefly outline the computational procedure to arrive at these results. First, to obtain the Feynman rules for the effective Lagrangian and the energy-momentum tensor, we used the program FeynCalc. To perform the calculations of the pertinent two-loop diagrams, we wrote a program in Mathematica which contracts the Lorentz indices in the products of the Feynman rules and expands the obtained expressions. This results in hundreds of thousands of terms. In these, we reduce the tensor integrals to the master scalar integrals specified in the Appendix. Next, the program substitutes the explicit expressions of the scalar integrals and simplifies the obtained result to very compact expressions as specified above. For obvious reasons, we do not display any of these intermediate steps here.

\section{IMPLICATION ON THE COSMOLOGICAL CONSTANT PROBLEM}

It follows from the result of the previous section that, unless the cosmological constant is chosen such that the energy of the vacuum is exactly zero, it cannot remove the graviton mass and the graviton tadpole order by order in perturbation theory, and consequently a nonperturbative treatment of the cosmological constant term is mandatory. This is because for all physical processes there appear diagrams like the ones shown in Fig. 4, where the massless graviton propagator carries vanishing momentum, and therefore 1/0 singularities occur (this does not happen only if the tadpole vanishes order by order in the loop expansion).

The cosmological constant problem is often described as a vacuum having tiny nonzero energy density. Because of this loose language, one might think that the condition of vanishing vacuum energy a priori excludes the solution of the cosmological constant problem. A closer look reveals that exactly the opposite might be the case. Indeed, because of the condition imposed on the cosmological constant term of the effective Lagrangian, the effective action calculated on the Minkowski background metric with vanishing background matter fields does not contain an effective cosmological constant term contributing to Einsten's equations. However, for our Universe the corresponding effective action has to be calculated in the presence of nontrivial background fields. The cosmological constant term of the

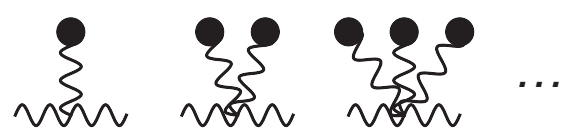

FIG. 4. Tree order tadpole diagrams contributing to the graviton self-energy. 
effective Lagrangian exactly canceling the loop contributions in a trivial background leads to a uniquely fixed effective cosmological constant contributing in the Einstein's equation also in the presence of a nontrivial background. While for weak backgrounds we expect large cancellations leaving us with a tiny effective cosmological constant, a quantitative investigation of this estimation is a subject for a separate publication. To make this more precise, the background relevant for cosmology is not flat Minkowski; therefore the fixed cosmological constant term will cancel the quantum corrections to the effective cosmological constant not exactly but rather only approximately, leaving a small finite piece. This remains to be calculated.

\section{SUMMARY AND DISCUSSION}

Consistency conditions of the perturbative EFT of general relativity in a flat Minkowski background uniquely fix the cosmological constant term as a function of all other parameters of the theory [6]. This follows from the requirement of the presence of a massless graviton, instead of a massive spin- 2 ghost, in the spectrum of the theory. Notice that it is not possible to take into account perturbatively any other value of the cosmological constant term within an EFT on the flat Minkowski background. This is because of the $1 / 0$ singularities in the Feynman diagrams with tadpole contributions; see, e.g., Fig. 4.

In our opinion if there is any fundamental reason for choosing a fixed value of the cosmological constant, then it must be the condition of vanishing of the vacuum energy. It is often argued that vacuum has nonzero energy due to quantum fluctuations. A classical example is given by quantum oscillator. It is well known that the ground state energy of a quantum oscillator is $\hbar \omega / 2$, where $\omega$ is the angular frequency. A closer look reveals, however, that this expression is the result of an assumption. In particular, if we share the point of view that the real world is described by a quantum theory and classical theory is only an approximation of it, then it is not possible to uniquely reproduce the quantum Hamiltonian of an oscillator by quantizing the classical one. This nonuniqueness is of course well known and is manifested in the problem of operator ordering. Indeed, by adding a vanishing term $\sim(p q-q p)$ to the classical Hamiltonian of the oscillator and quantizing it, we obtain a quantum Hamiltonian with an arbitrary constant term, and hence an arbitrary vacuum energy. Starting from the classical theory, there is no way to tell which value of the vacuum energy is more "fundamental." Notice that the argument for the Casimir effect being a proof of nonvanishing vacuum energy is not convincing either; see, e.g., Refs. [20,21].

In the framework of low-energy EFT of general relativity coupled to the fields of the Standard Model, imposing a condition of vanishing vacuum energy uniquely fixes the cosmological constant term as a function of other parameters of the effective Lagrangian. We expect that this will lead to a self-consistent perturbative EFT defined on the Minkowski background, i.e., to a massless graviton in the spectrum and the vanishing graviton tadpole. We were not able to give a general argument supporting our claim. Instead we calculated the vacuum expectation value of the full four-momentum of matter and gravitational fields at two-loop order in a simplified version of the Abelian model with spontaneous symmetry breaking considered in Ref. [6]. While at oneloop order the condition of vanishing vacuum energy automatically leads to the conditions of Ref. [6], at twoloop order the same agreement of two conditions appears to be a result of a nontrivial cancellation between different diagrams. We notice here that there does not exist a commonly accepted expression of the energy-momentum tensor for the gravitational field (see, e.g., Refs. [11-15]). In this work we used the definition of the energy-momentum pseudotensor and the full fourmomentum of the matter and gravitational fields given in the classic textbook by Landau and Lifshitz [17].

Within a self-consistent EFT all physical quantities should be finite after renormalizing (an infinite number of) parameters of the effective Lagrangian. Therefore it is mandatory that the uniquely fixed value of the cosmological constant term, which defines the perturbative EFT of the Standard Model coupled to gravitons on the Minkowski flat background leads to a finite expression of the energy of the vacuum to all orders in loop expansion. Based on the two-loop order result of this work, we expect that this finite value is actually zero. Turning the argument around, we expect that, by demanding that the vacuum energy should be vanishing to all orders, we obtain a self-consistent perturbative low-energy EFT of matter and gravitational fields on the flat Minkowski background.

Relegating calculations and detailed discussion to a future work, we briefly comment on the implications of our results for the cosmological constant problem. In particular, we expect the cosmological constant term of the effective Lagrangian exactly canceling the loop contributions in a flat background very likely to cancel the bulk of such contributions also in the presence of a nontrivial background, relevant for our Universe, thus leaving with a tiny effective cosmological constant contributing to Einstein's equations.

A further very interesting question is what condition (if any) is imposed on the energy of the vacuum by the consistency of EFT of gravitation in (anti-)de Sitter space. Unfortunately we are unable to address this issue as, to the best of our knowledge, a consistent formulation of a systematic EFT in the (anti-)de Sitter background does not exist yet. 


\section{ACKNOWLEDGMENTS}

We thank Dalibor Djukanovic for the helpful comments on the manuscript. We are also grateful to the referee for some pertinent remarks that helped to improve the manuscript. The work of J. G. was supported in part by BMBF (Grant No. 05P18PCFP1), and by the Georgian Shota Rustaveli National Science Foundation (Grant No. FR17354). The work of U.-G. M. was supported in part by provided by Deutsche Forschungsgemeinschaft (DFG) through funds provided to the Sino-German CRC 110, "Symmetries and the Emergence of Structure in QCD" (Grant No. TRR110), by the Chinese Academy of Sciences (CAS) through the President's International Fellowship Initiative (PIFI) (Grant No. 2018DM0034), and by the VolkswagenStiftung (Grant No. 93562).

\section{APPENDIX: FEYNMAN RULES}

Below we give Feynman rules used in the calculation of the vacuum expectation values of the graviton field and the energy-momentum tensor.

Propagators:

(a) Scalar propagator with momentum $p$ :

$$
\frac{i}{p^{2}-m^{2}+i \epsilon}
$$

(b) Vector boson propagator with Lorentz indices $\mu, \nu$ and momentum $p$ :

$$
-\frac{i\left(g^{\mu \nu}-p^{\mu} p^{\nu} / M^{2}\right)}{p^{2}-M^{2}+i \epsilon} .
$$

(c) Graviton propagator in $D$ dimensions with Lorentz indices $(\mu, \nu),(\alpha, \beta)$ and momentum $p$ :

$$
\begin{aligned}
& \frac{i g^{\lambda \nu} g^{\mu \sigma}+g^{\lambda \mu} g^{\nu \sigma}-\frac{2 g^{\lambda \sigma} g^{\mu \nu}}{D-2}}{p^{2}+i \epsilon} \\
& \quad-\frac{i \xi}{2} \frac{p^{\nu}\left(p^{\sigma} g^{\lambda \mu}+p^{\lambda} g^{\mu \sigma}\right)+p^{\mu}\left(p^{\sigma} g^{\lambda \nu}+p^{\lambda} g^{\nu \sigma}\right)}{\left(p^{2}+i \epsilon\right)^{2}}
\end{aligned}
$$

Vertices (all momenta in all vertices are incoming):

(a) Graviton with indices $(\mu, \nu)$ :

$$
-\frac{2 i \Lambda g^{\mu \nu}}{\kappa}
$$

(b) Graviton with indices $(\mu, \nu)$ and $(\alpha, \beta)$ :

$$
i \Lambda\left(g^{\alpha \nu} g^{\beta \mu}+g^{\alpha \mu} g^{\beta \nu}-g^{\alpha \beta} g^{\mu \nu}\right) .
$$

(c) Graviton with indices $(\mu, \nu)$ —scalars with momenta $p_{1}$ and $p_{2}$ :

$$
\frac{1}{2} i \kappa\left(-g^{\mu \nu}\left(m^{2}+p_{1} \cdot p_{2}\right)+p_{2}^{\mu} p_{1}^{\nu}+p_{1}^{\mu} p_{2}^{\nu}\right)
$$

(d) Gravitons with indices $(\mu, \nu)$ and $(\alpha, \beta)$-scalars with momenta $p_{1}$ and $p_{2}$ :

$$
\begin{aligned}
- & \frac{1}{4} i \kappa^{2}\left(-m^{2} g^{\alpha \nu} g^{\beta \mu}-m^{2} g^{\alpha \mu} g^{\beta \nu}+m^{2} g^{\alpha \beta} g^{\mu \nu}+p_{1}^{\beta} p_{2}^{\nu} g^{\alpha \mu}+p_{1}^{\beta} p_{2}^{\mu} g^{\alpha \nu}+p_{1}^{\alpha} p_{2}^{\nu} g^{\beta \mu}+p_{1}^{\nu}\left(-p_{2}^{\mu} g^{\alpha \beta}+p_{2}^{\beta} g^{\alpha \mu}+p_{2}^{\alpha} g^{\beta \mu}\right)\right. \\
& \left.+p_{1}^{\alpha} p_{2}^{\mu} g^{\beta \nu}+p_{1}^{\mu}\left(-p_{2}^{\nu} g^{\alpha \beta}+p_{2}^{\beta} g^{\alpha \nu}+p_{2}^{\alpha} g^{\beta \nu}\right)-p_{2}^{\alpha} p_{1}^{\beta} g^{\mu \nu}-p_{1}^{\alpha} p_{2}^{\beta} g^{\mu \nu}-p_{1} \cdot p_{2}\left(g^{\alpha \nu} g^{\beta \mu}+g^{\alpha \mu} g^{\beta \nu}-g^{\alpha \beta} g^{\mu \nu}\right)\right) .
\end{aligned}
$$

(e) Graviton with indices $(\mu, \nu)$ —vector bosons with (Lorentz index, momentum) combinations $\left(\lambda, p_{1}\right)$ and $\left(\sigma, p_{2}\right)$ :

$$
\begin{aligned}
- & \frac{i}{2} \kappa\left(-M^{2} g^{\lambda \sigma} g^{\mu \nu}+M^{2} g^{\lambda \nu} g^{\mu \sigma}+M^{2} g^{\lambda \mu} g^{\nu \sigma}+p_{1}^{\mu} p_{2}^{\nu} g^{\lambda \sigma}-p_{1}^{\sigma}\left(p_{2}^{\nu} g^{\lambda \mu}+p_{2}^{\mu} g^{\lambda \nu}-p_{2}^{\lambda} g^{\mu \nu}\right)\right. \\
& \left.+p_{1}^{\nu}\left(p_{2}^{\mu} g^{\lambda \sigma}-p_{2}^{\lambda} g^{\mu \sigma}\right)-p_{2}^{\lambda} p_{1}^{\mu} g^{\nu \sigma}-p_{1} \cdot p_{2} g^{\lambda \sigma} g^{\mu \nu}+p_{1} \cdot p_{2} g^{\lambda \nu} g^{\mu \sigma}+p_{1} \cdot p_{2} g^{\lambda \mu} g^{\nu \sigma}\right) .
\end{aligned}
$$


(f) Gravitons with indices $(\mu, \nu)$ and $(\alpha, \beta)$-vector bosons with (Lorentz index, momentum) combinations $\left(\lambda, p_{1}\right)$ and $\left(\sigma, p_{2}\right)$ :

$$
\begin{aligned}
& -\frac{i}{4} \kappa^{2}\left(-g^{\alpha \sigma} g^{\beta \nu} g^{\lambda \mu} M^{2}-g^{\alpha \nu} g^{\beta \sigma} g^{\lambda \mu} M^{2}-g^{\alpha \sigma} g^{\beta \mu} g^{\lambda \nu} M^{2}-g^{\alpha \mu} g^{\beta \sigma} g^{\lambda \nu} M^{2}+g^{\alpha \nu} g^{\beta \mu} g^{\lambda \sigma} M^{2}+g^{\alpha \mu} g^{\beta \nu} g^{\lambda \sigma} M^{2}+g^{\alpha \sigma} g^{\beta \lambda} g^{\mu \nu} M^{2}\right. \\
& +g^{\alpha \lambda} g^{\beta \sigma} g^{\mu \nu} M^{2}-g^{\alpha \beta} g^{\lambda \sigma} g^{\mu \nu} M^{2}-g^{\alpha \nu} g^{\beta \lambda} g^{\mu \sigma} M^{2}-g^{\alpha \lambda} g^{\beta \nu} g^{\mu \sigma} M^{2}+g^{\alpha \beta} g^{\lambda \nu} g^{\mu \sigma} M^{2}-g^{\alpha \mu} g^{\beta \lambda} g^{\nu \sigma} M^{2}-g^{\alpha \lambda} g^{\beta \mu} g^{\nu \sigma} M^{2} \\
& +g^{\alpha \beta} g^{\lambda \mu} g^{\nu \sigma} M^{2}-p_{1}^{\mu} p_{2}^{\nu} g^{\alpha \sigma} g^{\beta \lambda}+p_{1}^{\mu} p_{2}^{\lambda} g^{\alpha \sigma} g^{\beta \nu}-p_{1}^{\mu} p_{2}^{\nu} g^{\alpha \lambda} g^{\beta \sigma}+p_{1}^{\mu} p_{2}^{\lambda} g^{\alpha \nu} g^{\beta \sigma}+p_{1}^{\beta} p_{2}^{\nu} g^{\alpha \sigma} g^{\lambda \mu}+p_{1}^{\alpha} p_{2}^{\nu} g^{\beta \sigma} g^{\lambda \mu} \\
& +p_{1}^{\beta} p_{2}^{\mu} g^{\alpha \sigma} g^{\lambda \nu}+p_{1}^{\alpha} p_{2}^{\mu} g^{\beta \sigma} g^{\lambda \nu}+p_{1}^{\mu} p_{2}^{\nu} g^{\alpha \beta} g^{\lambda \sigma}-p_{1}^{\beta} p_{2}^{\nu} g^{\alpha \mu} g^{\lambda \sigma}-p_{1}^{\mu} p_{2}^{\beta} g^{\alpha \nu} g^{\lambda \sigma}-p_{1}^{\beta} p_{2}^{\mu} g^{\alpha \nu} g^{\lambda \sigma}-p_{1}^{\alpha} p_{2}^{\nu} g^{\beta \mu} g^{\lambda \sigma} \\
& -p_{1}^{\mu} p_{2}^{\alpha} g^{\beta \nu} g^{\lambda \sigma}-p_{1}^{\alpha} p_{2}^{\mu} g^{\beta \nu} g^{\lambda \sigma}-p_{1}^{\beta} p_{2}^{\lambda} g^{\alpha \sigma} g^{\mu \nu}-p_{1}^{\alpha} p_{2}^{\lambda} g^{\beta \sigma} g^{\mu \nu}+p_{1}^{\beta} p_{2}^{\alpha} g^{\lambda \sigma} g^{\mu \nu}+p_{1}^{\alpha} p_{2}^{\beta} g^{\lambda \sigma} g^{\mu \nu} \\
& +p_{1}^{\sigma}\left(p_{2}^{\mu} g^{\alpha \nu} g^{\beta \lambda}-p_{2}^{\alpha} g^{\mu \nu} g^{\beta \lambda}-p_{2}^{\lambda} g^{\alpha \nu} g^{\beta \mu}+p_{2}^{\mu} g^{\alpha \lambda} g^{\beta \nu}-p_{2}^{\lambda} g^{\alpha \mu} g^{\beta \nu}+p_{2}^{\alpha} g^{\beta \nu} g^{\lambda \mu}+p_{2}^{\nu}\left(g^{\alpha \mu} g^{\beta \lambda}+g^{\alpha \lambda} g^{\beta \mu}-g^{\alpha \beta} g^{\lambda \mu}\right)\right. \\
& \left.-p_{2}^{\mu} g^{\alpha \beta} g^{\lambda \nu}+p_{2}^{\alpha} g^{\beta \mu} g^{\lambda \nu}+p_{2}^{\lambda} g^{\alpha \beta} g^{\mu \nu}+p_{2}^{\beta}\left(g^{\alpha \nu} g^{\lambda \mu}+g^{\alpha \mu} g^{\lambda \nu}-g^{\alpha \lambda} g^{\mu \nu}\right)\right)+p_{1}^{\beta} p_{2}^{\lambda} g^{\alpha \nu} g^{\mu \sigma} \\
& +p_{1}^{\alpha} p_{2}^{\lambda} g^{\beta \nu} g^{\mu \sigma}-p_{1}^{\beta} p_{2}^{\alpha} g^{\lambda \nu} g^{\mu \sigma}-p_{1}^{\alpha} p_{2}^{\beta} g^{\lambda \nu} g^{\mu \sigma}+p_{1}^{\nu}\left(-p_{2}^{\mu} g^{\alpha \sigma} g^{\beta \lambda}+p_{2}^{\alpha} g^{\mu \sigma} g^{\beta \lambda}-p_{2}^{\mu} g^{\alpha \lambda} g^{\beta \sigma}+p_{2}^{\mu} g^{\alpha \beta} g^{\lambda \sigma}\right. \\
& \left.-p_{2}^{\alpha} g^{\beta \mu} g^{\lambda \sigma}+p_{2}^{\lambda}\left(g^{\alpha \sigma} g^{\beta \mu}+g^{\alpha \mu} g^{\beta \sigma}-g^{\alpha \beta} g^{\mu \sigma}\right)+p_{2}^{\beta}\left(g^{\alpha \lambda} g^{\mu \sigma}-g^{\alpha \mu} g^{\lambda \sigma}\right)\right)-p_{1}^{\mu} p_{2}^{\lambda} g^{\alpha \beta} g^{\nu \sigma} \\
& +p_{1}^{\mu} p_{2}^{\beta} g^{\alpha \lambda} g^{\nu \sigma}+p_{1}^{\beta} p_{2}^{\lambda} g^{\alpha \mu} g^{\nu \sigma}+p_{1}^{\mu} p_{2}^{\alpha} g^{\beta \lambda} g^{\nu \sigma}+p_{1}^{\alpha} p_{2}^{\lambda} g^{\beta \mu} g^{\nu \sigma}-p_{1}^{\beta} p_{2}^{\alpha} g^{\lambda \mu} g^{\nu \sigma}-p_{1}^{\alpha} p_{2}^{\beta} g^{\lambda \mu} g^{\nu \sigma} \\
& -g^{\alpha \sigma} g^{\beta \nu} g^{\lambda \mu} p_{1} \cdot p_{2}-g^{\alpha \nu} g^{\beta \sigma} g^{\lambda \mu} p_{1} \cdot p_{2}-g^{\alpha \sigma} g^{\beta \mu} g^{\lambda \nu} p_{1} \cdot p_{2}-g^{\alpha \mu} g^{\beta \sigma} g^{\lambda \nu} p_{1} \cdot p_{2} \\
& +g^{\alpha \nu} g^{\beta \mu} g^{\lambda \sigma} p_{1} \cdot p_{2}+g^{\alpha \mu} g^{\beta \nu} g^{\lambda \sigma} p_{1} \cdot p_{2}+g^{\alpha \sigma} g^{\beta \lambda} g^{\mu \nu} p_{1} \cdot p_{2}+g^{\alpha \lambda} g^{\beta \sigma} g^{\mu \nu} p_{1} \cdot p_{2}-g^{\alpha \beta} g^{\lambda \sigma} g^{\mu \nu} p_{1} \cdot p_{2} \\
& -g^{\alpha \nu} g^{\beta \lambda} g^{\mu \sigma} p_{1} \cdot p_{2}-g^{\alpha \lambda} g^{\beta \nu} g^{\mu \sigma} p_{1} \cdot p_{2}+g^{\alpha \beta} g^{\lambda \nu} g^{\mu \sigma} p_{1} \cdot p_{2}-g^{\alpha \mu} g^{\beta \lambda} g^{\nu \sigma} p_{1} \cdot p_{2}-g^{\alpha \lambda} g^{\beta \mu} g^{\nu \sigma} p_{1} \cdot p_{2} \\
& \left.+g^{\alpha \beta} g^{\lambda \mu} g^{\nu \sigma} p_{1} \cdot p_{2}\right) \text {. }
\end{aligned}
$$

(g) Energy-momentum tensor with indices $(\mu, \nu)$-gravitons with (Lorentz indices, momentum) combinations $\left(\lambda, \sigma, p_{1}\right)$ and $\left(\alpha, \beta, p_{2}\right)$ :

$$
\begin{aligned}
\frac{1}{8} & \operatorname{hhh}\left(\left\{\mu, \nu, p_{1}\right\},\left\{\alpha, \beta, p_{2}\right\},\left\{\lambda, \sigma, p_{3}\right\}\right)+\operatorname{hhh}\left(\left\{\mu, \nu, p_{1}\right\},\left\{\alpha, \beta, p_{2}\right\},\left\{\sigma, \lambda, p_{3}\right\}\right) \\
& +\operatorname{hhh}\left(\left\{\mu, \nu, p_{1}\right\},\left\{\beta, \alpha, p_{2}\right\},\left\{\lambda, \sigma, p_{3}\right\}\right)+\operatorname{hhh}\left(\left\{\mu, \nu, p_{1}\right\},\left\{\beta, \alpha, p_{2}\right\},\left\{\sigma, \lambda, p_{3}\right\}\right) \\
& +\operatorname{hhh}\left(\left\{\nu, \mu, p_{1}\right\},\left\{\alpha, \beta, p_{2}\right\},\left\{\lambda, \sigma, p_{3}\right\}\right)+\operatorname{hhh}\left(\left\{\nu, \mu, p_{1}\right\},\left\{\alpha, \beta, p_{2}\right\},\left\{\sigma, \lambda, p_{3}\right\}\right) \\
& \left.+\operatorname{hhh}\left(\left\{\nu, \mu, p_{1}\right\},\left\{\beta, \alpha, p_{2}\right\},\left\{\lambda, \sigma, p_{3}\right\}\right)+\operatorname{hhh}\left(\left\{\nu, \mu, p_{1}\right\},\left\{\beta, \alpha, p_{2}\right\},\left\{\sigma, \lambda, p_{3}\right\}\right)\right],
\end{aligned}
$$

where

$$
\begin{aligned}
& \operatorname{hhh}\left(\left\{\mu, \nu, p_{1}\right\},\left\{\alpha, \beta, p_{2}\right\},\left\{\lambda, \sigma, p_{3}\right\}\right) \\
& =-\frac{1}{4} i \kappa\left(p_{1}^{\alpha} p_{2}^{\beta} g^{\lambda \sigma} g^{\mu \nu}+p_{2}^{\beta} p_{3}^{\alpha} g^{\lambda \sigma} g^{\mu \nu}+2 \Lambda g^{\alpha \beta} g^{\lambda \sigma} g^{\mu \nu}+2 g^{\alpha \beta} g^{\lambda \sigma}\left(p_{1} \cdot p_{2}+p_{1} \cdot p_{3}+p_{2} \cdot p_{3}\right) g^{\mu \nu}+2 g^{\alpha \beta} g^{\lambda \sigma}\left(p_{1}^{2}+p_{2}^{2}+p_{3}^{2}\right) g^{\mu \nu}\right. \\
& +g^{\alpha \beta}\left(p_{1}^{\nu}\left(p_{2}^{\mu}+p_{3}^{\mu}\right) g^{\lambda \sigma}+\left(p_{1}^{\lambda}+p_{2}^{\lambda}\right) p_{3}^{\sigma} g^{\mu \nu}\right)+4\left(p_{1}^{\sigma} p_{3}^{\nu} g^{\alpha \beta} g^{\lambda \mu}+p_{1}^{\beta} p_{2}^{\nu} g^{\alpha \mu} g^{\lambda \sigma}+p_{2}^{\sigma} p_{3}^{\beta} g^{\alpha \lambda} g^{\mu \nu}\right) \\
& +4\left(\left(p_{1}^{\sigma} p_{2}^{\nu}+p_{2}^{\sigma} p_{3}^{\nu}\right) g^{\alpha \beta} g^{\lambda \mu}+\left(p_{2}^{\nu} p_{3}^{\beta}+p_{1}^{\beta} p_{3}^{\nu}\right) g^{\alpha \mu} g^{\lambda \sigma}+\left(p_{1}^{\beta} p_{2}^{\sigma}+p_{1}^{\sigma} p_{3}^{\beta}\right) g^{\alpha \lambda} g^{\mu \nu}\right) \\
& -2\left(\left(p_{1}^{\nu} p_{2}^{\sigma}+p_{2}^{\nu} p_{3}^{\sigma}\right) g^{\alpha \beta} g^{\lambda \mu}+\left(p_{1}^{\nu} p_{3}^{\beta}+p_{2}^{\beta} p_{3}^{\nu}\right) g^{\alpha \mu} g^{\lambda \sigma}+\left(p_{1}^{\sigma} p_{2}^{\beta}+p_{1}^{\beta} p_{3}^{\sigma}\right) g^{\alpha \lambda} g^{\mu \nu}\right) \\
& +2\left(\left(p_{1}^{\nu} p_{1}^{\sigma}+p_{3}^{\nu} p_{3}^{\sigma}\right) g^{\alpha \beta} g^{\lambda \mu}+\left(p_{1}^{\beta} p_{1}^{\nu}+p_{2}^{\beta} p_{2}^{\nu}\right) g^{\alpha \mu} g^{\lambda \sigma}+\left(p_{2}^{\beta} p_{2}^{\sigma}+p_{3}^{\beta} p_{3}^{\sigma}\right) g^{\alpha \lambda} g^{\mu \nu}\right) \\
& -2\left(p_{1}^{\mu} p_{1}^{\nu} g^{\alpha \beta} g^{\lambda \sigma}+\left(p_{3}^{\lambda} p_{3}^{\sigma} g^{\alpha \beta}+p_{2}^{\alpha} p_{2}^{\beta} g^{\lambda \sigma}\right) g^{\mu \nu}\right)-2\left(p_{1}^{\beta} p_{3}^{\alpha} g^{\lambda \sigma} g^{\mu \nu}+p_{1}^{\alpha} p_{3}^{\beta} g^{\lambda \sigma} g^{\mu \nu}\right. \\
& \left.+g^{\alpha \beta}\left(\left(p_{2}^{\nu} p_{3}^{\mu}+p_{2}^{\mu} p_{3}^{\nu}\right) g^{\lambda \sigma}+\left(p_{1}^{\sigma} p_{2}^{\lambda}+p_{1}^{\lambda} p_{2}^{\sigma}\right) g^{\mu \nu}\right)\right)-4\left(p_{1}^{\alpha} p_{1}^{\beta} g^{\lambda \sigma} g^{\mu \nu}+p_{3}^{\alpha} p_{3}^{\beta} g^{\lambda \sigma} g^{\mu \nu}\right. \\
& \left.+g^{\alpha \beta}\left(\left(p_{2}^{\mu} p_{2}^{\nu}+p_{3}^{\mu} p_{3}^{\nu}\right) g^{\lambda \sigma}+\left(p_{1}^{\lambda} p_{1}^{\sigma}+p_{2}^{\lambda} p_{2}^{\sigma}\right) g^{\mu \nu}\right)\right)-5\left(p_{2}^{\alpha}\left(p_{1}^{\beta}+p_{3}^{\beta}\right) g^{\lambda \sigma} g^{\mu \nu}+g^{\alpha \beta}\left(p_{1}^{\mu}\left(p_{2}^{\nu}+p_{3}^{\nu}\right) g^{\lambda \sigma}\right.\right. \\
& \left.\left.+\left(p_{1}^{\sigma}+p_{2}^{\sigma}\right) p_{3}^{\lambda} g^{\mu \nu}\right)\right)+2\left(p_{3}^{\mu} p_{3}^{\sigma} g^{\alpha \beta} g^{\lambda \nu}+p_{1}^{\alpha} p_{1}^{\nu} g^{\beta \mu} g^{\lambda \sigma}+p_{3}^{\alpha} p_{3}^{\sigma} g^{\beta \lambda} g^{\mu \nu}+p_{2}^{\beta}\left(p_{2}^{\mu} g^{\alpha \nu} g^{\lambda \sigma}+p_{2}^{\lambda} g^{\alpha \sigma} g^{\mu \nu}\right)\right. \\
& \left.+p_{1}^{\lambda} p_{1}^{\nu} g^{\alpha \beta} g^{\mu \sigma}\right)+2\left(p_{2}^{\sigma} p_{3}^{\mu} g^{\alpha \beta} g^{\lambda \nu}+p_{2}^{\mu} p_{3}^{\beta} g^{\alpha \nu} g^{\lambda \sigma}+p_{1}^{\alpha} p_{3}^{\nu} g^{\beta \mu} g^{\lambda \sigma}+p_{1}^{\beta} p_{2}^{\lambda} g^{\alpha \sigma} g^{\mu \nu}+p_{1}^{\sigma} p_{3}^{\alpha} g^{\beta \lambda} g^{\mu \nu}+p_{1}^{\lambda} p_{2}^{\nu} g^{\alpha \beta} g^{\mu \sigma}\right) \\
& +4\left(p_{1}^{\sigma} p_{3}^{\mu} g^{\alpha \beta} g^{\lambda \nu}+p_{1}^{\beta} p_{2}^{\mu} g^{\alpha \nu} g^{\lambda \sigma}+p_{1}^{\alpha} p_{2}^{\nu} g^{\beta \mu} g^{\lambda \sigma}+p_{2}^{\lambda} p_{3}^{\beta} g^{\alpha \sigma} g^{\mu \nu}+p_{2}^{\sigma} p_{3}^{\alpha} g^{\beta \lambda} g^{\mu \nu}+p_{1}^{\lambda} p_{3}^{\nu} g^{\alpha \beta} g^{\mu \sigma}\right)
\end{aligned}
$$


$-4\left(p_{1}^{\beta} p_{3}^{\nu} g^{\alpha \sigma} g^{\lambda \mu}+p_{1}^{\sigma}\left(p_{2}^{\nu} g^{\alpha \mu} g^{\beta \lambda}+p_{3}^{\beta} g^{\alpha \nu} g^{\lambda \mu}\right)+p_{2}^{\sigma}\left(p_{3}^{\nu} g^{\alpha \lambda} g^{\beta \mu}+p_{1}^{\beta} g^{\alpha \mu} g^{\lambda \nu}\right)+p_{2}^{\nu} p_{3}^{\beta} g^{\alpha \lambda} g^{\mu \sigma}\right)$

$-6\left(p_{2}^{\nu} p_{3}^{\beta} g^{\alpha \sigma} g^{\lambda \mu}+p_{2}^{\sigma}\left(p_{3}^{\nu} g^{\alpha \mu} g^{\beta \lambda}+p_{1}^{\beta} g^{\alpha \nu} g^{\lambda \mu}\right)+p_{1}^{\sigma}\left(p_{2}^{\nu} g^{\alpha \lambda} g^{\beta \mu}+p_{3}^{\beta} g^{\alpha \mu} g^{\lambda \nu}\right)+p_{1}^{\beta} p_{3}^{\nu} g^{\alpha \lambda} g^{\mu \sigma}\right)$

$+8 \Lambda\left(g^{\alpha \sigma} g^{\beta \mu} g^{\lambda \nu}+g^{\alpha \nu} g^{\beta \lambda} g^{\mu \sigma}\right)-2\left(p_{2}^{\nu} p_{3}^{\mu} g^{\alpha \sigma} g^{\beta \lambda}+p_{2}^{\mu} p_{3}^{\nu} g^{\alpha \sigma} g^{\beta \lambda}+p_{1}^{\sigma} p_{2}^{\lambda} g^{\alpha \nu} g^{\beta \mu}+p_{1}^{\lambda} p_{2}^{\sigma} g^{\alpha \nu} g^{\beta \mu}\right.$

$\left.+p_{1}^{\beta} p_{3}^{\alpha} g^{\lambda \nu} g^{\mu \sigma}+p_{1}^{\alpha} p_{3}^{\beta} g^{\lambda \nu} g^{\mu \sigma}\right)-2\left(p_{1}^{\sigma} p_{3}^{\alpha} g^{\beta \mu} g^{\lambda \nu}+g^{\alpha \sigma}\left(p_{1}^{\lambda} p_{2}^{\nu} g^{\beta \mu}+p_{2}^{\mu} p_{3}^{\beta} g^{\lambda \nu}\right)+p_{1}^{\alpha} p_{3}^{\nu} g^{\beta \lambda} g^{\mu \sigma}\right.$

$\left.+g^{\alpha \nu}\left(p_{2}^{\sigma} p_{3}^{\mu} g^{\beta \lambda}+p_{1}^{\beta} p_{2}^{\lambda} g^{\mu \sigma}\right)\right)+2\left(p_{2}^{\nu} p_{3}^{\sigma} g^{\alpha \mu} g^{\beta \lambda}+p_{1}^{\sigma} p_{2}^{\beta} g^{\alpha \nu} g^{\lambda \mu}+p_{2}^{\beta} p_{3}^{\nu} g^{\alpha \sigma} g^{\lambda \mu}+p_{1}^{\beta} p_{3}^{\sigma} g^{\alpha \mu} g^{\lambda \nu}\right.$

$\left.+p_{1}^{\nu} g^{\alpha \lambda}\left(p_{2}^{\sigma} g^{\beta \mu}+p_{3}^{\beta} g^{\mu \sigma}\right)\right)+2\left(p_{1}^{\mu}\left(p_{2}^{\sigma} g^{\alpha \beta} g^{\lambda \nu}+p_{3}^{\beta} g^{\alpha \nu} g^{\lambda \sigma}\right)+p_{2}^{\alpha}\left(p_{3}^{\nu} g^{\beta \mu} g^{\lambda \sigma}+p_{1}^{\sigma} g^{\beta \lambda} g^{\mu \nu}\right)\right.$

$\left.+p_{3}^{\lambda}\left(p_{1}^{\beta} g^{\alpha \sigma} g^{\mu \nu}+p_{2}^{\nu} g^{\alpha \beta} g^{\mu \sigma}\right)\right)-2\left(p_{1}^{\mu}\left(p_{3}^{\sigma} g^{\alpha \beta} g^{\lambda \nu}+p_{2}^{\beta} g^{\alpha \nu} g^{\lambda \sigma}\right)+\left(p_{2}^{\beta} p_{3}^{\lambda} g^{\alpha \sigma}+p_{2}^{\alpha} p_{3}^{\sigma} g^{\beta \lambda}\right) g^{\mu \nu}\right.$

$\left.+p_{1}^{\nu}\left(p_{2}^{\alpha} g^{\beta \mu} g^{\lambda \sigma}+p_{3}^{\lambda} g^{\alpha \beta} g^{\mu \sigma}\right)\right)+2\left(p_{1}^{\mu}\left(p_{1}^{\sigma} g^{\alpha \beta} g^{\lambda \nu}+p_{1}^{\beta} g^{\alpha \nu} g^{\lambda \sigma}\right)+p_{2}^{\alpha}\left(p_{2}^{\nu} g^{\beta \mu} g^{\lambda \sigma}+p_{2}^{\sigma} g^{\beta \lambda} g^{\mu \nu}\right)\right.$

$\left.+p_{3}^{\lambda}\left(p_{3}^{\beta} g^{\alpha \sigma} g^{\mu \nu}+p_{3}^{\nu} g^{\alpha \beta} g^{\mu \sigma}\right)\right)-4\left(p_{1}^{\sigma} p_{3}^{\nu}\left(g^{\alpha \mu} g^{\beta \lambda}+g^{\alpha \lambda} g^{\beta \mu}\right)+p_{2}^{\sigma} p_{3}^{\beta}\left(g^{\alpha \nu} g^{\lambda \mu}+g^{\alpha \mu} g^{\lambda \nu}\right)\right.$

$\left.+p_{1}^{\beta} p_{2}^{\nu}\left(g^{\alpha \sigma} g^{\lambda \mu}+g^{\alpha \lambda} g^{\mu \sigma}\right)\right)-4\left(p_{2}^{\nu} p_{2}^{\sigma}\left(g^{\alpha \mu} g^{\beta \lambda}+g^{\alpha \lambda} g^{\beta \mu}\right)+p_{1}^{\beta} p_{1}^{\sigma}\left(g^{\alpha \nu} g^{\lambda \mu}+g^{\alpha \mu} g^{\lambda \nu}\right)\right.$

$\left.+p_{3}^{\beta} p_{3}^{\nu}\left(g^{\alpha \sigma} g^{\lambda \mu}+g^{\alpha \lambda} g^{\mu \sigma}\right)\right)+2\left(p_{2}^{\mu} p_{3}^{\sigma} g^{\alpha \nu} g^{\beta \lambda}+p_{2}^{\beta} p_{3}^{\mu} g^{\alpha \sigma} g^{\lambda \nu}+p_{1}^{\alpha} p_{3}^{\sigma} g^{\beta \mu} g^{\lambda \nu}+p_{1}^{\lambda} p_{2}^{\beta} g^{\alpha \nu} g^{\mu \sigma}\right.$

$\left.+p_{1}^{\nu}\left(p_{2}^{\lambda} g^{\alpha \sigma} g^{\beta \mu}+p_{3}^{\alpha} g^{\beta \lambda} g^{\mu \sigma}\right)\right)+16\left(p_{3}^{\alpha} p_{3}^{\mu} g^{\beta \nu} g^{\lambda \sigma}+p_{1}^{\alpha} p_{1}^{\lambda} g^{\beta \sigma} g^{\mu \nu}+p_{2}^{\lambda} p_{2}^{\mu} g^{\alpha \beta} g^{\nu \sigma}\right)-8\left(p_{1}^{\alpha} p_{2}^{\mu} g^{\beta \nu} g^{\lambda \sigma}\right.$

$\left.+p_{2}^{\lambda} p_{3}^{\alpha} g^{\beta \sigma} g^{\mu \nu}+p_{1}^{\lambda} p_{3}^{\mu} g^{\alpha \beta} g^{\nu \sigma}\right)+2\left(p_{1}^{\alpha} p_{1}^{\mu} g^{\beta \nu} g^{\lambda \sigma}+p_{3}^{\alpha} p_{3}^{\lambda} g^{\beta \sigma} g^{\mu \nu}+p_{2}^{\alpha}\left(p_{2}^{\mu} g^{\beta \nu} g^{\lambda \sigma}+p_{2}^{\lambda} g^{\beta \sigma} g^{\mu \nu}\right)\right.$

$\left.+p_{1}^{\lambda} p_{1}^{\mu} g^{\alpha \beta} g^{\nu \sigma}+p_{3}^{\lambda} p_{3}^{\mu} g^{\alpha \beta} g^{\nu \sigma}\right)+2\left(p_{3}^{\alpha}\left(p_{2}^{\mu} g^{\beta \nu} g^{\lambda \sigma}+p_{1}^{\lambda} g^{\beta \sigma} g^{\mu \nu}\right)+p_{1}^{\alpha}\left(p_{3}^{\mu} g^{\beta \nu} g^{\lambda \sigma}+p_{2}^{\lambda} g^{\beta \sigma} g^{\mu \nu}\right)\right.$

$\left.+\left(p_{1}^{\lambda} p_{2}^{\mu}+p_{2}^{\lambda} p_{3}^{\mu}\right) g^{\alpha \beta} g^{\nu \sigma}\right)-4\left(p_{2}^{\mu} p_{3}^{\lambda} g^{\alpha \sigma} g^{\beta \nu}+p_{1}^{\alpha} p_{3}^{\lambda} g^{\mu \sigma} g^{\beta \nu}+p_{1}^{\mu} g^{\beta \sigma}\left(p_{2}^{\lambda} g^{\alpha \nu}+p_{3}^{\alpha} g^{\lambda \nu}\right)+p_{2}^{\alpha} p_{3}^{\mu} g^{\beta \lambda} g^{\nu \sigma}\right.$

$\left.+p_{1}^{\lambda} p_{2}^{\alpha} g^{\beta \mu} g^{\nu \sigma}\right)-8\left(p_{1}^{\lambda} p_{1}^{\mu} g^{\alpha \nu} g^{\beta \sigma}+p_{1}^{\alpha} p_{1}^{\mu} g^{\lambda \nu} g^{\beta \sigma}+p_{3}^{\lambda} g^{\beta \nu}\left(p_{3}^{\mu} g^{\alpha \sigma}+p_{3}^{\alpha} g^{\mu \sigma}\right)+p_{2}^{\alpha} p_{2}^{\mu} g^{\beta \lambda} g^{\nu \sigma}+p_{2}^{\alpha} p_{2}^{\lambda} g^{\beta \mu} g^{\nu \sigma}\right)$

$+4\left(p_{3}^{\lambda} p_{3}^{\sigma} g^{\alpha \mu} g^{\beta \nu}+p_{1}^{\mu} p_{1}^{\nu} g^{\alpha \lambda} g^{\beta \sigma}+p_{2}^{\alpha} p_{2}^{\beta} g^{\lambda \mu} g^{\nu \sigma}\right)-2\left(p_{1}^{\lambda} p_{3}^{\sigma} g^{\alpha \mu} g^{\beta \nu}+p_{2}^{\lambda} p_{3}^{\sigma} g^{\alpha \mu} g^{\beta \nu}+p_{1}^{\nu} p_{2}^{\mu} g^{\alpha \lambda} g^{\beta \sigma}\right.$

$\left.+p_{1}^{\nu} p_{3}^{\mu} g^{\alpha \lambda} g^{\beta \sigma}+p_{1}^{\alpha} p_{2}^{\beta} g^{\lambda \mu} g^{\nu \sigma}+p_{2}^{\beta} p_{3}^{\alpha} g^{\lambda \mu} g^{\nu \sigma}\right)+8\left(p_{1}^{\sigma} p_{2}^{\lambda} g^{\alpha \mu} g^{\beta \nu}+p_{1}^{\lambda} p_{2}^{\sigma} g^{\alpha \mu} g^{\beta \nu}+p_{2}^{\nu} p_{3}^{\mu} g^{\alpha \lambda} g^{\beta \sigma}+p_{2}^{\mu} p_{3}^{\nu} g^{\alpha \lambda} g^{\beta \sigma}\right.$

$\left.+p_{1}^{\beta} p_{3}^{\alpha} g^{\lambda \mu} g^{\nu \sigma}+p_{1}^{\alpha} p_{3}^{\beta} g^{\lambda \mu} g^{\nu \sigma}\right)+8\left(p_{1}^{\lambda} p_{1}^{\sigma} g^{\alpha \mu} g^{\beta \nu}+p_{2}^{\lambda} p_{2}^{\sigma} g^{\alpha \mu} g^{\beta \nu}+p_{2}^{\mu} p_{2}^{\nu} g^{\alpha \lambda} g^{\beta \sigma}+p_{3}^{\mu} p_{3}^{\nu} g^{\alpha \lambda} g^{\beta \sigma}+p_{1}^{\alpha} p_{1}^{\beta} g^{\lambda \mu} g^{\nu \sigma}\right.$

$\left.+p_{3}^{\alpha} p_{3}^{\beta} g^{\lambda \mu} g^{\nu \sigma}\right)+10\left(\left(p_{1}^{\sigma}+p_{2}^{\sigma}\right) p_{3}^{\lambda} g^{\alpha \mu} g^{\beta \nu}+p_{1}^{\mu}\left(p_{2}^{\nu}+p_{3}^{\nu}\right) g^{\alpha \lambda} g^{\beta \sigma}+p_{2}^{\alpha}\left(p_{1}^{\beta}+p_{3}^{\beta}\right) g^{\lambda \mu} g^{\nu \sigma}\right)$

$-4 \Lambda\left(g^{\alpha \mu} g^{\beta \nu} g^{\lambda \sigma}+g^{\alpha \lambda} g^{\beta \sigma} g^{\mu \nu}+g^{\alpha \beta} g^{\lambda \mu} g^{\nu \sigma}\right)+4\left(p_{2}^{\alpha}\left(p_{3}^{\sigma} g^{\beta \nu}+p_{1}^{\nu} g^{\beta \sigma}\right) g^{\lambda \mu}+p_{1}^{\mu} g^{\alpha \lambda}\left(p_{3}^{\sigma} g^{\beta \nu}+p_{2}^{\beta} g^{\nu \sigma}\right)\right.$

$\left.+p_{3}^{\lambda} g^{\alpha \mu}\left(p_{1}^{\nu} g^{\beta \sigma}+p_{2}^{\beta} g^{\nu \sigma}\right)\right)-8\left(\left(p_{3}^{\alpha} p_{3}^{\sigma} g^{\beta \nu}+p_{1}^{\alpha} p_{1}^{\nu} g^{\beta \sigma}\right) g^{\lambda \mu}+g^{\alpha \mu}\left(p_{1}^{\lambda} p_{1}^{\nu} g^{\beta \sigma}+p_{2}^{\beta} p_{2}^{\lambda} g^{\nu \sigma}\right)\right.$

$\left.+g^{\alpha \lambda}\left(p_{3}^{\mu} p_{3}^{\sigma} g^{\beta \nu}+p_{2}^{\beta} p_{2}^{\mu} g^{\nu \sigma}\right)\right)-10\left(p_{2}^{\alpha}\left(p_{1}^{\sigma} g^{\beta \nu}+p_{3}^{\nu} g^{\beta \sigma}\right) g^{\lambda \mu}+p_{3}^{\lambda} g^{\alpha \mu}\left(p_{2}^{\nu} g^{\beta \sigma}+p_{1}^{\beta} g^{\nu \sigma}\right)\right.$

$\left.+p_{1}^{\mu} g^{\alpha \lambda}\left(p_{2}^{\sigma} g^{\beta \nu}+p_{3}^{\beta} g^{\nu \sigma}\right)\right)-4\left(\left(p_{2}^{\sigma} p_{3}^{\alpha} g^{\beta \nu}+p_{1}^{\alpha} p_{2}^{\nu} g^{\beta \sigma}\right) g^{\lambda \mu}+g^{\alpha \lambda}\left(p_{1}^{\sigma} p_{3}^{\mu} g^{\beta \nu}+p_{1}^{\beta} p_{2}^{\mu} g^{\nu \sigma}\right)\right.$

$\left.+g^{\alpha \mu}\left(p_{1}^{\lambda} p_{3}^{\nu} g^{\beta \sigma}+p_{2}^{\lambda} p_{3}^{\beta} g^{\nu \sigma}\right)\right)-4\left(\left(p_{1}^{\sigma} p_{3}^{\alpha} g^{\beta \nu}+p_{1}^{\alpha} p_{3}^{\nu} g^{\beta \sigma}\right) g^{\lambda \mu}+g^{\alpha \mu}\left(p_{1}^{\lambda} p_{2}^{\nu} g^{\beta \sigma}+p_{1}^{\beta} p_{2}^{\lambda} g^{\nu \sigma}\right)\right.$

$\left.+g^{\alpha \lambda}\left(p_{2}^{\sigma} p_{3}^{\mu} g^{\beta \nu}+p_{2}^{\mu} p_{3}^{\beta} g^{\nu \sigma}\right)\right)+10\left(\left(p_{1}^{\alpha} p_{2}^{\sigma} g^{\beta \nu}+p_{2}^{\nu} p_{3}^{\alpha} g^{\beta \sigma}\right) g^{\lambda \mu}+g^{\alpha \mu}\left(p_{2}^{\lambda} p_{3}^{\nu} g^{\beta \sigma}+p_{1}^{\lambda} p_{3}^{\beta} g^{\nu \sigma}\right)\right.$

$\left.+g^{\alpha \lambda}\left(p_{1}^{\sigma} p_{2}^{\mu} g^{\beta \nu}+p_{1}^{\beta} p_{3}^{\mu} g^{\nu \sigma}\right)\right)+8\left(p_{2}^{\alpha}\left(p_{3}^{\mu} g^{\beta \nu} g^{\lambda \sigma}+p_{1}^{\lambda} g^{\beta \sigma} g^{\mu \nu}\right)+p_{1}^{\mu}\left(p_{3}^{\alpha} g^{\beta \nu} g^{\lambda \sigma}+p_{2}^{\lambda} g^{\alpha \beta} g^{\nu \sigma}\right)\right.$

$\left.+p_{3}^{\lambda}\left(p_{1}^{\alpha} g^{\beta \sigma} g^{\mu \nu}+p_{2}^{\mu} g^{\alpha \beta} g^{\nu \sigma}\right)\right)+12\left(p_{2}^{\alpha} p_{3}^{\lambda} g^{\beta \sigma} g^{\mu \nu}+p_{1}^{\mu}\left(p_{2}^{\alpha} g^{\beta \nu} g^{\lambda \sigma}+p_{3}^{\lambda} g^{\alpha \beta} g^{\nu \sigma}\right)\right)-6\left(p_{2}^{\mu} p_{3}^{\lambda} g^{\alpha \nu} g^{\beta \sigma}\right.$

$\left.+p_{2}^{\alpha} p_{3}^{\mu} g^{\lambda \nu} g^{\beta \sigma}+p_{1}^{\lambda} p_{2}^{\alpha} g^{\beta \nu} g^{\mu \sigma}+p_{1}^{\alpha} p_{3}^{\lambda} g^{\beta \mu} g^{\nu \sigma}+p_{1}^{\mu}\left(p_{2}^{\lambda} g^{\alpha \sigma} g^{\beta \nu}+p_{3}^{\alpha} g^{\beta \lambda} g^{\nu \sigma}\right)\right)-4\left(p_{2}^{\lambda} p_{2}^{\mu}\left(g^{\alpha \sigma} g^{\beta \nu}+g^{\alpha \nu} g^{\beta \sigma}\right)\right.$

$\left.+p_{3}^{\alpha} p_{3}^{\mu}\left(g^{\beta \sigma} g^{\lambda \nu}+g^{\beta \lambda} g^{\nu \sigma}\right)+p_{1}^{\alpha} p_{1}^{\lambda}\left(g^{\beta \nu} g^{\mu \sigma}+g^{\beta \mu} g^{\nu \sigma}\right)\right)+4\left(p_{1}^{\lambda} p_{3}^{\mu}\left(g^{\alpha \sigma} g^{\beta \nu}+g^{\alpha \nu} g^{\beta \sigma}\right)\right.$

$\left.+p_{1}^{\alpha} p_{2}^{\mu}\left(g^{\beta \sigma} g^{\lambda \nu}+g^{\beta \lambda} g^{\nu \sigma}\right)+p_{2}^{\lambda} p_{3}^{\alpha}\left(g^{\beta \nu} g^{\mu \sigma}+g^{\beta \mu} g^{\nu \sigma}\right)\right)-4\left(p_{1}^{\alpha} p_{3}^{\mu} g^{\beta \sigma} g^{\lambda \nu}+p_{1}^{\lambda}\left(p_{2}^{\mu} g^{\alpha \nu} g^{\beta \sigma}+p_{3}^{\alpha} g^{\beta \nu} g^{\mu \sigma}\right)\right.$

$\left.+p_{2}^{\mu} p_{3}^{\alpha} g^{\beta \lambda} g^{\nu \sigma}+p_{2}^{\lambda}\left(p_{3}^{\mu} g^{\alpha \sigma} g^{\beta \nu}+p_{1}^{\alpha} g^{\beta \mu} g^{\nu \sigma}\right)\right)+2\left(p_{2}^{\mu} p_{3}^{\alpha} g^{\beta \sigma} g^{\lambda \nu}+p_{2}^{\lambda}\left(p_{3}^{\mu} g^{\alpha \nu} g^{\beta \sigma}+p_{1}^{\alpha} g^{\beta \nu} g^{\mu \sigma}\right)+p_{1}^{\alpha} p_{3}^{\mu} g^{\beta \lambda} g^{\nu \sigma}\right.$

$\left.+p_{1}^{\lambda}\left(p_{2}^{\mu} g^{\alpha \sigma} g^{\beta \nu}+p_{3}^{\alpha} g^{\beta \mu} g^{\nu \sigma}\right)\right)-12\left(p_{2}^{\alpha} p_{3}^{\lambda}\left(g^{\beta \nu} g^{\mu \sigma}+g^{\beta \mu} g^{\nu \sigma}\right)+p_{1}^{\mu}\left(p_{3}^{\lambda}\left(g^{\alpha \sigma} g^{\beta \nu}+g^{\alpha \nu} g^{\beta \sigma}\right)+p_{2}^{\alpha}\left(g^{\beta \sigma} g^{\lambda \nu}+g^{\beta \lambda} g^{\nu \sigma}\right)\right)\right)$

$+4\left(g^{\alpha \sigma} g^{\beta \nu} g^{\lambda \mu} p_{1} \cdot p_{2}+g^{\alpha \mu} g^{\beta \lambda} g^{\nu \sigma} p_{1} \cdot p_{3}+g^{\alpha \lambda}\left(g^{\beta \nu} g^{\mu \sigma} p_{1} \cdot p_{2}+g^{\beta \mu} g^{\nu \sigma} p_{1} \cdot p_{3}\right)\right.$

$\left.+g^{\alpha \nu} g^{\beta \sigma} g^{\lambda \mu} p_{2} \cdot p_{3}+g^{\alpha \mu} g^{\beta \sigma} g^{\lambda \nu} p_{2} \cdot p_{3}\right)+2\left(g^{\alpha \nu} g^{\beta \mu} g^{\lambda \sigma} p_{1} \cdot p_{2}+g^{\alpha \beta} g^{\lambda \nu} g^{\mu \sigma} p_{1} \cdot p_{3}+g^{\alpha \sigma} g^{\beta \lambda} g^{\mu \nu} p_{2} \cdot p_{3}\right)$

$-8\left(g^{\alpha \mu} g^{\beta \nu} g^{\lambda \sigma} p_{1} \cdot p_{2}+g^{\alpha \beta} g^{\lambda \mu} g^{\nu \sigma} p_{1} \cdot p_{3}+g^{\alpha \lambda} g^{\beta \sigma} g^{\mu \nu} p_{2} \cdot p_{3}\right)+8\left(g^{\alpha \mu}\left(g^{\beta \sigma} g^{\lambda \nu}\right.\right.$ 


$$
\begin{aligned}
& \left.\left.+g^{\beta \lambda} g^{\nu \sigma}\right) p_{1} \cdot p_{2}+g^{\alpha \sigma} g^{\beta \nu} g^{\lambda \mu} p_{1} \cdot p_{3}+g^{\alpha \nu} g^{\beta \sigma} g^{\lambda \mu} p_{1} \cdot p_{3}+g^{\alpha \lambda} g^{\beta \nu} g^{\mu \sigma} p_{2} \cdot p_{3}+g^{\alpha \lambda} g^{\beta \mu} g^{\nu \sigma} p_{2} \cdot p_{3}\right) \\
& -4\left(g^{\alpha \lambda} g^{\beta \sigma} g^{\mu \nu}\left(p_{1} \cdot p_{2}+p_{1} \cdot p_{3}\right)+g^{\alpha \beta} g^{\lambda \mu} g^{\nu \sigma}\left(p_{1} \cdot p_{2}+p_{2} \cdot p_{3}\right)+g^{\alpha \mu} g^{\beta \nu} g^{\lambda \sigma}\left(p_{1} \cdot p_{3}+p_{2} \cdot p_{3}\right)\right) \\
& -4\left(g^{\alpha \lambda} g^{\beta \sigma} g^{\mu \nu} p_{1}^{2}+g^{\alpha \beta} g^{\lambda \mu} g^{\nu \sigma} p_{2}^{2}+g^{\alpha \mu} g^{\beta \nu} g^{\lambda \sigma} p_{3}^{2}\right)+8\left(g^{\alpha \nu} g^{\beta \sigma} g^{\lambda \mu} p_{1}^{2}+g^{\alpha \lambda} g^{\beta \mu} g^{\nu \sigma} p_{2}^{2}+g^{\alpha \sigma} g^{\beta \nu} g^{\lambda \mu} p_{3}^{2}\right. \\
& \left.+g^{\alpha \lambda} g^{\beta \nu} g^{\mu \sigma} p_{3}^{2}+g^{\alpha \mu}\left(g^{\beta \sigma} g^{\lambda \nu} p_{1}^{2}+g^{\beta \lambda} g^{\nu \sigma} p_{2}^{2}\right)\right)-4\left(g^{\alpha \mu} g^{\beta \nu} g^{\lambda \sigma}\left(p_{1}^{2}+p_{2}^{2}\right)\right. \\
& \left.\left.+g^{\alpha \beta} g^{\lambda \mu} g^{\nu \sigma}\left(p_{1}^{2}+p_{3}^{2}\right)+g^{\alpha \lambda} g^{\beta \sigma} g^{\mu \nu}\left(p_{2}^{2}+p_{3}^{2}\right)\right)\right) .
\end{aligned}
$$

(h) Energy-momentum tensor with indices $(\mu, \nu)$ - scalars with momenta $p_{1}$ and $p_{2}$ :

$$
g^{\mu \nu}\left(m^{2}+p_{1} \cdot p_{2}\right)-p_{2}^{\mu} p_{1}^{\nu}-p_{1}^{\mu} p_{2}^{\nu} .
$$

(i) Energy-momentum tensor with indices $(\mu, \nu)$-graviton with indices $(\alpha, \beta)$ and scalars with momenta $p_{1}$ and $p_{2}$ :

$$
\begin{aligned}
\frac{1}{2} & \kappa\left(m^{2}\left(-g^{\alpha \nu}\right) g^{\beta \mu}-m^{2} g^{\alpha \mu} g^{\beta \nu}+2 m^{2} g^{\alpha \beta} g^{\mu \nu}+p_{1}^{\beta} p_{2}^{\nu} g^{\alpha \mu}+p_{1}^{\beta} p_{2}^{\mu} g^{\alpha \nu}+p_{1}^{\alpha} p_{2}^{\nu} g^{\beta \mu}\right. \\
& +p_{1}^{\nu}\left(-2 p_{2}^{\mu} g^{\alpha \beta}+p_{2}^{\beta} g^{\alpha \mu}+p_{2}^{\alpha} g^{\beta \mu}\right)+p_{1}^{\alpha} p_{2}^{\mu} g^{\beta \nu}+p_{1}^{\mu}\left(-2 p_{2}^{\nu} g^{\alpha \beta}+p_{2}^{\beta} g^{\alpha \nu}+p_{2}^{\alpha} g^{\beta \nu}\right)-p_{2}^{\alpha} p_{1}^{\beta} g^{\mu \nu} \\
& \left.-p_{1}^{\alpha} p_{2}^{\beta} g^{\mu \nu}-p_{1} \cdot p_{2} g^{\alpha \nu} g^{\beta \mu}-p_{1} \cdot p_{2} g^{\alpha \mu} g^{\beta \nu}+2 p_{1} \cdot p_{2} g^{\alpha \beta} g^{\mu \nu}\right) .
\end{aligned}
$$

(j) Energy-momentum tensor with indices $(\mu, \nu)$-vector bosons with (Lorentz index, momentum) combinations $\left(\lambda, p_{1}\right)$ and $\left(\sigma, p_{2}\right)$ :

$$
\begin{aligned}
- & M^{2} g^{\lambda \sigma} g^{\mu \nu}+M^{2} g^{\lambda \nu} g^{\mu \sigma}+M^{2} g^{\lambda \mu} g^{\nu \sigma}+p_{1}^{\mu} p_{2}^{\nu} g^{\lambda \sigma}-p_{1}^{\sigma}\left(p_{2}^{\nu} g^{\lambda \mu}+p_{2}^{\mu} g^{\lambda \nu}-p_{2}^{\lambda} g^{\mu \nu}\right) \\
& +p_{1}^{\nu}\left(p_{2}^{\mu} g^{\lambda \sigma}-p_{2}^{\lambda} g^{\mu \sigma}\right)-p_{2}^{\lambda} p_{1}^{\mu} g^{\nu \sigma}-p_{1} \cdot p_{2} g^{\lambda \sigma} g^{\mu \nu}+p_{1} \cdot p_{2} g^{\lambda \nu} g^{\mu \sigma}+p_{1} \cdot p_{2} g^{\lambda \mu} g^{\nu \sigma} .
\end{aligned}
$$

(k) Energy-momentum tensor with indices $(\mu, \nu)$-graviton with indices $(\alpha, \beta)$ and vector bosons with (Lorentz index, momentum) combinations $\left(\lambda, p_{1}\right)$ and $\left(\sigma, p_{2}\right)$ :

$$
\begin{aligned}
& -\frac{1}{2} \kappa\left(g^{\alpha \sigma} g^{\beta \nu} g^{\lambda \mu} M^{2}+g^{\alpha \nu} g^{\beta \sigma} g^{\lambda \mu} M^{2}+g^{\alpha \sigma} g^{\beta \mu} g^{\lambda \nu} M^{2}+g^{\alpha \mu} g^{\beta \sigma} g^{\lambda \nu} M^{2}-g^{\alpha \nu} g^{\beta \mu} g^{\lambda \sigma} M^{2}-g^{\alpha \mu} g^{\beta \nu} g^{\lambda \sigma} M^{2}\right. \\
& -g^{\alpha \sigma} g^{\beta \lambda} g^{\mu \nu} M^{2}-g^{\alpha \lambda} g^{\beta \sigma} g^{\mu \nu} M^{2}+2 g^{\alpha \beta} g^{\lambda \sigma} g^{\mu \nu} M^{2}+g^{\alpha \nu} g^{\beta \lambda} g^{\mu \sigma} M^{2}+g^{\alpha \lambda} g^{\beta \nu} g^{\mu \sigma} M^{2}-2 g^{\alpha \beta} g^{\lambda \nu} g^{\mu \sigma} M^{2} \\
& +g^{\alpha \mu} g^{\beta \lambda} g^{\nu \sigma} M^{2}+g^{\alpha \lambda} g^{\beta \mu} g^{\nu \sigma} M^{2}-2 g^{\alpha \beta} g^{\lambda \mu} g^{\nu \sigma} M^{2}+p_{1}^{\mu} p_{2}^{\nu} g^{\alpha \sigma} g^{\beta \lambda}-p_{1}^{\mu} p_{2}^{\lambda} g^{\alpha \sigma} g^{\beta \nu}+p_{1}^{\mu} p_{2}^{\nu} g^{\alpha \lambda} g^{\beta \sigma} \\
& -p_{1}^{\mu} p_{2}^{\lambda} g^{\alpha \nu} g^{\beta \sigma}-p_{1}^{\beta} p_{2}^{\nu} g^{\alpha \sigma} g^{\lambda \mu}-p_{1}^{\alpha} p_{2}^{\nu} g^{\beta \sigma} g^{\lambda \mu}-p_{1}^{\beta} p_{2}^{\mu} g^{\alpha \sigma} g^{\lambda \nu}-p_{1}^{\alpha} p_{2}^{\mu} g^{\beta \sigma} g^{\lambda \nu}-2 p_{1}^{\mu} p_{2}^{\nu} g^{\alpha \beta} g^{\lambda \sigma}+p_{1}^{\beta} p_{2}^{\nu} g^{\alpha \mu} g^{\lambda \sigma} \\
& +p_{1}^{\mu} p_{2}^{\beta} g^{\alpha \nu} g^{\lambda \sigma}+p_{1}^{\beta} p_{2}^{\mu} g^{\alpha \nu} g^{\lambda \sigma}+p_{1}^{\alpha} p_{2}^{\nu} g^{\beta \mu} g^{\lambda \sigma}+p_{1}^{\mu} p_{2}^{\alpha} g^{\beta \nu} g^{\lambda \sigma}+p_{1}^{\alpha} p_{2}^{\mu} g^{\beta \nu} g^{\lambda \sigma}+p_{1}^{\beta} p_{2}^{\lambda} g^{\alpha \sigma} g^{\mu \nu}+p_{1}^{\alpha} p_{2}^{\lambda} g^{\beta \sigma} g^{\mu \nu} \\
& -p_{1}^{\beta} p_{2}^{\alpha} g^{\lambda \sigma} g^{\mu \nu}-p_{1}^{\alpha} p_{2}^{\beta} g^{\lambda \sigma} g^{\mu \nu}-p_{1}^{\sigma}\left(-p_{2}^{\lambda} g^{\alpha \nu} g^{\beta \mu}+p_{2}^{\alpha} g^{\lambda \nu} g^{\beta \mu}-p_{2}^{\lambda} g^{\alpha \mu} g^{\beta \nu}+p_{2}^{\beta} g^{\alpha \nu} g^{\lambda \mu}+p_{2}^{\alpha} g^{\beta \nu} g^{\lambda \mu}\right. \\
& +p_{2}^{\nu}\left(g^{\alpha \mu} g^{\beta \lambda}+g^{\alpha \lambda} g^{\beta \mu}-2 g^{\alpha \beta} g^{\lambda \mu}\right)+p_{2}^{\beta} g^{\alpha \mu} g^{\lambda \nu}+p_{2}^{\mu}\left(g^{\alpha \nu} g^{\beta \lambda}+g^{\alpha \lambda} g^{\beta \nu}-2 g^{\alpha \beta} g^{\lambda \nu}\right)+2 p_{2}^{\lambda} g^{\alpha \beta} g^{\mu \nu} \\
& \left.-p_{2}^{\beta} g^{\alpha \lambda} g^{\mu \nu}-p_{2}^{\alpha} g^{\beta \lambda} g^{\mu \nu}\right)-p_{1}^{\beta} p_{2}^{\lambda} g^{\alpha \nu} g^{\mu \sigma}-p_{1}^{\alpha} p_{2}^{\lambda} g^{\beta \nu} g^{\mu \sigma}+p_{1}^{\beta} p_{2}^{\alpha} g^{\lambda \nu} g^{\mu \sigma}+p_{1}^{\alpha} p_{2}^{\beta} g^{\lambda \nu} g^{\mu \sigma} \\
& +p_{1}^{\nu}\left(p_{2}^{\beta} g^{\alpha \mu} g^{\lambda \sigma}+p_{2}^{\alpha} g^{\beta \mu} g^{\lambda \sigma}+p_{2}^{\mu}\left(g^{\alpha \sigma} g^{\beta \lambda}+g^{\alpha \lambda} g^{\beta \sigma}-2 g^{\alpha \beta} g^{\lambda \sigma}\right)-p_{2}^{\beta} g^{\alpha \lambda} g^{\mu \sigma}-p_{2}^{\alpha} g^{\beta \lambda} g^{\mu \sigma}\right. \\
& \left.+p_{2}^{\lambda}\left(-g^{\alpha \sigma} g^{\beta \mu}-g^{\alpha \mu} g^{\beta \sigma}+2 g^{\alpha \beta} g^{\mu \sigma}\right)\right)+2 p_{1}^{\mu} p_{2}^{\lambda} g^{\alpha \beta} g^{\nu \sigma}-p_{1}^{\mu} p_{2}^{\beta} g^{\alpha \lambda} g^{\nu \sigma}-p_{1}^{\beta} p_{2}^{\lambda} g^{\alpha \mu} g^{\nu \sigma}-p_{1}^{\mu} p_{2}^{\alpha} g^{\beta \lambda} g^{\nu \sigma} \\
& -p_{1}^{\alpha} p_{2}^{\lambda} g^{\beta \mu} g^{\nu \sigma}+p_{1}^{\beta} p_{2}^{\alpha} g^{\lambda \mu} g^{\nu \sigma}+p_{1}^{\alpha} p_{2}^{\beta} g^{\lambda \mu} g^{\nu \sigma}+g^{\alpha \sigma} g^{\beta \nu} g^{\lambda \mu} p_{1} \cdot p_{2}+g^{\alpha \nu} g^{\beta \sigma} g^{\lambda \mu} p_{1} \cdot p_{2}+g^{\alpha \sigma} g^{\beta \mu} g^{\lambda \nu} p_{1} \cdot p_{2} \\
& +g^{\alpha \mu} g^{\beta \sigma} g^{\lambda \nu} p_{1} \cdot p_{2}-g^{\alpha \nu} g^{\beta \mu} g^{\lambda \sigma} p_{1} \cdot p_{2}-g^{\alpha \mu} g^{\beta \nu} g^{\lambda \sigma} p_{1} \cdot p_{2}-g^{\alpha \sigma} g^{\beta \lambda} g^{\mu \nu} p_{1} \cdot p_{2}-g^{\alpha \lambda} g^{\beta \sigma} g^{\mu \nu} p_{1} \cdot p_{2} \\
& +2 g^{\alpha \beta} g^{\lambda \sigma} g^{\mu \nu} p_{1} \cdot p_{2}+g^{\alpha \nu} g^{\beta \lambda} g^{\mu \sigma} p_{1} \cdot p_{2}+g^{\alpha \lambda} g^{\beta \nu} g^{\mu \sigma} p_{1} \cdot p_{2}-2 g^{\alpha \beta} g^{\lambda \nu} g^{\mu \sigma} p_{1} \cdot p_{2} \\
& \left.+g^{\alpha \mu} g^{\beta \lambda} g^{\nu \sigma} p_{1} \cdot p_{2}+g^{\alpha \lambda} g^{\beta \mu} g^{\nu \sigma} p_{1} \cdot p_{2}-2 g^{\alpha \beta} g^{\lambda \mu} g^{\nu \sigma} p_{1} \cdot p_{2}\right) \text {. }
\end{aligned}
$$

(1) Energy-momentum tensor with indices $(\mu, \nu)$ —gravitons with (Lorentz indices, momentum) combinations $\left(\lambda, \sigma, p_{1}\right)$ and $\left(\alpha, \beta, p_{2}\right)$ : 


$$
\begin{aligned}
& \frac{1}{8}\left(-4 p_{1}^{\sigma} p_{2}^{\lambda} g^{\alpha \nu} g^{\beta \mu}-4 p_{1}^{\lambda} p_{2}^{\sigma} g^{\alpha \nu} g^{\beta \mu}+4 p_{1}^{\sigma} p_{2}^{\alpha} g^{\lambda \nu} g^{\beta \mu}-2 p_{1}^{\alpha} p_{2}^{\nu} g^{\lambda \sigma} g^{\beta \mu}-2 p_{1}^{\alpha} p_{2}^{\nu} g^{\sigma \lambda} g^{\beta \mu}+4 p_{1}^{\lambda} p_{2}^{\alpha} g^{\sigma \nu} g^{\beta \mu}\right. \\
& -4 g^{\alpha \sigma} g^{\lambda \nu} p_{1} \cdot p_{2} g^{\beta \mu}+6 g^{\alpha \nu} g^{\lambda \sigma} p_{1} \cdot p_{2} g^{\beta \mu}+6 g^{\alpha \nu} g^{\sigma \lambda} p_{1} \cdot p_{2} g^{\beta \mu}-4 g^{\alpha \lambda} g^{\sigma \nu} p_{1} \cdot p_{2} g^{\beta \mu}-4 p_{1}^{\sigma} p_{2}^{\lambda} g^{\alpha \mu} g^{\beta \nu} \\
& -4 p_{1}^{\lambda} p_{2}^{\sigma} g^{\alpha \mu} g^{\beta \nu}-4 p_{1}^{\sigma} p_{2}^{\nu} g^{\alpha \beta} g^{\lambda \mu}+4 p_{1}^{\sigma} p_{2}^{\beta} g^{\alpha \nu} g^{\lambda \mu}+4 p_{1}^{\beta} p_{2}^{\nu} g^{\alpha \sigma} g^{\lambda \mu}-4 p_{1}^{\sigma} p_{2}^{\nu} g^{\beta \alpha} g^{\lambda \mu}+4 p_{1}^{\sigma} p_{2}^{\alpha} g^{\beta \nu} g^{\lambda \mu} \\
& +4 p_{1}^{\alpha} p_{2}^{\nu} g^{\beta \sigma} g^{\lambda \mu}-4 p_{1}^{\sigma} p_{2}^{\mu} g^{\alpha \beta} g^{\lambda \nu}+4 p_{1}^{\sigma} p_{2}^{\beta} g^{\alpha \mu} g^{\lambda \nu}+4 p_{1}^{\beta} p_{2}^{\mu} g^{\alpha \sigma} g^{\lambda \nu}-4 p_{1}^{\sigma} p_{2}^{\mu} g^{\beta \alpha} g^{\lambda \nu}+4 p_{1}^{\alpha} p_{2}^{\mu} g^{\beta \sigma} g^{\lambda \nu} \\
& -2 p_{1}^{\beta} p_{2}^{\nu} g^{\alpha \mu} g^{\lambda \sigma}-2 p_{1}^{\beta} p_{2}^{\mu} g^{\alpha \nu} g^{\lambda \sigma}-2 p_{1}^{\alpha} p_{2}^{\mu} g^{\beta \nu} g^{\lambda \sigma}+2 p_{1}^{\sigma} p_{2}^{\lambda} g^{\alpha \beta} g^{\mu \nu}+2 p_{1}^{\lambda} p_{2}^{\sigma} g^{\alpha \beta} g^{\mu \nu}-2 p_{1}^{\beta} p_{2}^{\sigma} g^{\alpha \lambda} g^{\mu \nu} \\
& -2 p_{1}^{\beta} p_{2}^{\lambda} g^{\alpha \sigma} g^{\mu \nu}+2 p_{1}^{\sigma} p_{2}^{\lambda} g^{\beta \alpha} g^{\mu \nu}+2 p_{1}^{\lambda} p_{2}^{\sigma} g^{\beta \alpha} g^{\mu \nu}-2 p_{1}^{\alpha} p_{2}^{\sigma} g^{\beta \lambda} g^{\mu \nu}-2 p_{1}^{\alpha} p_{2}^{\lambda} g^{\beta \sigma} g^{\mu \nu}+2 p_{1}^{\beta} p_{2}^{\alpha} g^{\lambda \sigma} g^{\mu \nu} \\
& +2 p_{1}^{\alpha} p_{2}^{\beta} g^{\lambda \sigma} g^{\mu \nu}-2 p_{1}^{\beta} p_{2}^{\alpha} g^{\lambda \nu} g^{\mu \sigma}-2 p_{1}^{\alpha} p_{2}^{\beta} g^{\lambda \nu} g^{\mu \sigma}+2 p_{1}^{\sigma} p_{2}^{\lambda} g^{\alpha \beta} g^{\nu \mu}+2 p_{1}^{\lambda} p_{2}^{\sigma} g^{\alpha \beta} g^{\nu \mu}-2 p_{1}^{\beta} p_{2}^{\sigma} g^{\alpha \lambda} g^{\nu \mu} \\
& -2 p_{1}^{\beta} p_{2}^{\lambda} g^{\alpha \sigma} g^{\nu \mu}+2 p_{1}^{\sigma} p_{2}^{\lambda} g^{\beta \alpha} g^{\nu \mu}+2 p_{1}^{\lambda} p_{2}^{\sigma} g^{\beta \alpha} g^{\nu \mu}-2 p_{1}^{\alpha} p_{2}^{\sigma} g^{\beta \lambda} g^{\nu \mu}-2 p_{1}^{\alpha} p_{2}^{\lambda} g^{\beta \sigma} g^{\nu \mu}+2 p_{1}^{\beta} p_{2}^{\alpha} g^{\lambda \sigma} g^{\nu \mu} \\
& +2 p_{1}^{\alpha} p_{2}^{\beta} g^{\lambda \sigma} g^{\nu \mu}-2 p_{1}^{\beta} p_{2}^{\alpha} g^{\lambda \mu} g^{\nu \sigma}-2 p_{1}^{\alpha} p_{2}^{\beta} g^{\lambda \mu} g^{\nu \sigma}-2 p_{1}^{\beta} p_{2}^{\nu} g^{\alpha \mu} g^{\sigma \lambda}-2 p_{1}^{\beta} p_{2}^{\mu} g^{\alpha \nu} g^{\sigma \lambda}-2 p_{1}^{\alpha} p_{2}^{\mu} g^{\beta \nu} g^{\sigma \lambda} \\
& +2 p_{1}^{\beta} p_{2}^{\alpha} g^{\mu \nu} g^{\sigma \lambda}+2 p_{1}^{\alpha} p_{2}^{\beta} g^{\mu \nu} g^{\sigma \lambda}+2 p_{1}^{\beta} p_{2}^{\alpha} g^{\nu \mu} g^{\sigma \lambda}+2 p_{1}^{\alpha} p_{2}^{\beta} g^{\nu \mu} g^{\sigma \lambda}-4 p_{1}^{\lambda} p_{2}^{\nu} g^{\alpha \beta} g^{\sigma \mu}+4 p_{1}^{\beta} p_{2}^{\nu} g^{\alpha \lambda} g^{\sigma \mu} \\
& +4 p_{1}^{\lambda} p_{2}^{\beta} g^{\alpha \nu} g^{\sigma \mu}-4 p_{1}^{\lambda} p_{2}^{\nu} g^{\beta \alpha} g^{\sigma \mu}+4 p_{1}^{\alpha} p_{2}^{\nu} g^{\beta \lambda} g^{\sigma \mu}+4 p_{1}^{\lambda} p_{2}^{\alpha} g^{\beta \nu} g^{\sigma \mu}-2 p_{1}^{\beta} p_{2}^{\alpha} g^{\nu \lambda} g^{\sigma \mu}-2 p_{1}^{\alpha} p_{2}^{\beta} g^{\nu \lambda} g^{\sigma \mu} \\
& -2 p_{1}^{\nu}\left(-2 p_{2}^{\lambda} g^{\alpha \sigma} g^{\beta \mu}+2 p_{2}^{\alpha} g^{\lambda \sigma} g^{\beta \mu}+2 p_{2}^{\alpha} g^{\sigma \lambda} g^{\beta \mu}-2 p_{2}^{\lambda} g^{\alpha \mu} g^{\beta \sigma}\right. \\
& +p_{2}^{\sigma}\left(-2 g^{\alpha \mu} g^{\beta \lambda}-2 g^{\alpha \lambda} g^{\beta \mu}+\left(g^{\alpha \beta}+g^{\beta \alpha}\right) g^{\lambda \mu}\right)+2 p_{2}^{\beta} g^{\alpha \mu} g^{\lambda \sigma}+2 p_{2}^{\beta} g^{\alpha \mu} g^{\sigma \lambda} \\
& \left.+2 p_{2}^{\mu}\left(g^{\alpha \sigma} g^{\beta \lambda}+g^{\alpha \lambda} g^{\beta \sigma}-\left(g^{\alpha \beta}+g^{\beta \alpha}\right)\left(g^{\lambda \sigma}+g^{\sigma \lambda}\right)\right)+p_{2}^{\lambda} g^{\alpha \beta} g^{\sigma \mu}+p_{2}^{\lambda} g^{\beta \alpha} g^{\sigma \mu}\right)-4 p_{1}^{\lambda} p_{2}^{\mu} g^{\alpha \beta} g^{\sigma \nu} \\
& +4 p_{1}^{\beta} p_{2}^{\mu} g^{\alpha \lambda} g^{\sigma \nu}+4 p_{1}^{\lambda} p_{2}^{\beta} g^{\alpha \mu} g^{\sigma \nu}-4 p_{1}^{\lambda} p_{2}^{\mu} g^{\beta \alpha} g^{\sigma \nu}+4 p_{1}^{\alpha} p_{2}^{\mu} g^{\beta \lambda} g^{\sigma \nu}-2 p_{1}^{\beta} p_{2}^{\alpha} g^{\mu \lambda} g^{\sigma \nu}-2 p_{1}^{\alpha} p_{2}^{\beta} g^{\mu \lambda} g^{\sigma \nu} \\
& -2 p_{1}^{\mu}\left(-2 p_{2}^{\lambda} g^{\alpha \sigma} g^{\beta \nu}+2 p_{2}^{\alpha} g^{\lambda \sigma} g^{\beta \nu}+2 p_{2}^{\alpha} g^{\sigma \lambda} g^{\beta \nu}-2 p_{2}^{\lambda} g^{\alpha \nu} g^{\beta \sigma}\right. \\
& +p_{2}^{\sigma}\left(-2 g^{\alpha \nu} g^{\beta \lambda}-2 g^{\alpha \lambda} g^{\beta \nu}+\left(g^{\alpha \beta}+g^{\beta \alpha}\right) g^{\lambda \nu}\right)+2 p_{2}^{\beta} g^{\alpha \nu} g^{\lambda \sigma}+2 p_{2}^{\beta} g^{\alpha \nu} g^{\sigma \lambda} \\
& \left.+2 p_{2}^{\nu}\left(g^{\alpha \sigma} g^{\beta \lambda}+g^{\alpha \lambda} g^{\beta \sigma}-\left(g^{\alpha \beta}+g^{\beta \alpha}\right)\left(g^{\lambda \sigma}+g^{\sigma \lambda}\right)\right)+p_{2}^{\lambda} g^{\alpha \beta} g^{\sigma \nu}+p_{2}^{\lambda} g^{\beta \alpha} g^{\sigma \nu}\right)-4 g^{\alpha \sigma} g^{\beta \nu} g^{\lambda \mu} p_{1} \cdot p_{2} \\
& -4 g^{\alpha \nu} g^{\beta \sigma} g^{\lambda \mu} p_{1} \cdot p_{2}-4 g^{\alpha \mu} g^{\beta \sigma} g^{\lambda \nu} p_{1} \cdot p_{2}+6 g^{\alpha \mu} g^{\beta \nu} g^{\lambda \sigma} p_{1} \cdot p_{2}+2 g^{\alpha \sigma} g^{\beta \lambda} g^{\mu \nu} p_{1} \cdot p_{2}+2 g^{\alpha \lambda} g^{\beta \sigma} g^{\mu \nu} p_{1} \cdot p_{2} \\
& -3 g^{\alpha \beta} g^{\lambda \sigma} g^{\mu \nu} p_{1} \cdot p_{2}-3 g^{\beta \alpha} g^{\lambda \sigma} g^{\mu \nu} p_{1} \cdot p_{2}+3 g^{\alpha \beta} g^{\lambda \nu} g^{\mu \sigma} p_{1} \cdot p_{2}+3 g^{\beta \alpha} g^{\lambda \nu} g^{\mu \sigma} p_{1} \cdot p_{2}+2 g^{\alpha \sigma} g^{\beta \lambda} g^{\nu \mu} p_{1} \cdot p_{2} \\
& +2 g^{\alpha \lambda} g^{\beta \sigma} g^{\nu \mu} p_{1} \cdot p_{2}-3 g^{\alpha \beta} g^{\lambda \sigma} g^{\nu \mu} p_{1} \cdot p_{2}-3 g^{\beta \alpha} g^{\lambda \sigma} g^{\nu \mu} p_{1} \cdot p_{2}+3 g^{\alpha \beta} g^{\lambda \mu} g^{\nu \sigma} p_{1} \cdot p_{2}+3 g^{\beta \alpha} g^{\lambda \mu} g^{\nu \sigma} p_{1} \cdot p_{2} \\
& +6 g^{\alpha \mu} g^{\beta \nu} g^{\sigma \lambda} p_{1} \cdot p_{2}-3 g^{\alpha \beta} g^{\mu \nu} g^{\sigma \lambda} p_{1} \cdot p_{2}-3 g^{\beta \alpha} g^{\mu \nu} g^{\sigma \lambda} p_{1} \cdot p_{2}-3 g^{\alpha \beta} g^{\nu \mu} g^{\sigma \lambda} p_{1} \cdot p_{2}-3 g^{\beta \alpha} g^{\nu \mu} g^{\sigma \lambda} p_{1} \cdot p_{2} \\
& -4 g^{\alpha \nu} g^{\beta \lambda} g^{\sigma \mu} p_{1} \cdot p_{2}-4 g^{\alpha \lambda} g^{\beta \nu} g^{\sigma \mu} p_{1} \cdot p_{2}+3 g^{\alpha \beta} g^{\nu \lambda} g^{\sigma \mu} p_{1} \cdot p_{2}+3 g^{\beta \alpha} g^{\nu \lambda} g^{\sigma \mu} p_{1} \cdot p_{2}-4 g^{\alpha \mu} g^{\beta \lambda} g^{\sigma \nu} p_{1} \cdot p_{2} \\
& \left.+3 g^{\alpha \beta} g^{\mu \lambda} g^{\sigma \nu} p_{1} \cdot p_{2}+3 g^{\beta \alpha} g^{\mu \lambda} g^{\sigma \nu} p_{1} \cdot p_{2}\right) \text {. }
\end{aligned}
$$

The basic two-loop integral appearing in the results of the various two-loop diagrams takes the form

$$
\begin{aligned}
\int \frac{d^{n} k_{1} d^{n} k_{2}}{(2 \pi)^{2 n}} \frac{1}{\left(k_{1}^{2}-M^{2}+i \epsilon\right)^{\alpha}\left(k_{2}^{2}-M^{2}+i \epsilon\right)^{\beta}\left(\left(k_{1}-k_{2}\right)^{2}+i \epsilon\right)^{\gamma}} \\
=\frac{i^{2-2 \alpha-2 \beta-2 \gamma} M^{2(n-\alpha-\beta-\gamma)} \Gamma\left(\frac{n}{2}-\gamma\right) \Gamma\left(-\frac{n}{2}+\alpha+\gamma\right) \Gamma\left(-\frac{n}{2}+\beta+\gamma\right) \Gamma(-n+\alpha+\beta+\gamma)}{(4 \pi)^{n} \Gamma(\alpha) \Gamma(\beta) \Gamma\left(\frac{n}{2}\right) \Gamma(-n+\alpha+\beta+2 \gamma)} .
\end{aligned}
$$


[1] S. Weinberg, The Quantum Theory of Fields, Volumes 1 and 2 (Cambridge University Press, Cambridge, England, 2005).

[2] J.F. Donoghue, General relativity as an effective field theory: The leading quantum corrections, Phys. Rev. D 50, 3874 (1994).

[3] J. F. Donoghue and B. R. Holstein, Low energy theorems of quantum gravity from effective field theory, J. Phys. G $\mathbf{4 2}$, 103102 (2015).

[4] M. J. G. Veltman, Quantum theory of gravitation, Conf. Proc. C7507281, 265 (1975).

[5] J. F. Donoghue, M. M. Ivanov, and A. Shkerin, EPFL lectures on general relativity as a quantum field theory, arXiv: 1702.00319.

[6] D. Burns and A. Pilaftsis, Matter quantum corrections to the graviton self-energy and the Newtonian potential, Phys. Rev. D 91, 064047 (2015).

[7] S. Weinberg, The cosmological constant problem, Rev. Mod. Phys. 61, 1 (1989).

[8] J. Martin, Everything you always wanted to know about the cosmological constant problem (but were afraid to ask), C.R. Phys. 13, 566 (2012).

[9] G. Gabadadze and A. Gruzinov, Graviton mass or cosmological constant?, Phys. Rev. D 72, 124007 (2005).

[10] D. Rubin and B. Hayden, Is the expansion of the Universe accelerating? All signs point to yes, Astrophys. J. 833, L30 (2016).

[11] S. V. Babak and L. P. Grishchuk, Energy-momentum tensor for the gravitational field, Phys. Rev. D 61, 024038 (1999).
[12] L. M. Butcher, A. Lasenby, and M. Hobson, Physical significance of the Babak-Grishchuk gravitational energy-momentum tensor, Phys. Rev. D 78, 064034 (2008).

[13] L. B. Szabados, Quasi-local energy-momentum and angular momentum in general relativity, Living Rev. Relativity 12, 4 (2009).

[14] L. M. Butcher, M. Hobson, and A. Lasenby, Localizing the energy and momentum of linear gravity, Phys. Rev. D 82, 104040 (2010).

[15] L. M. Butcher, M. Hobson, and A. Lasenby, Localized energetics of linear gravity: Theoretical development, Phys. Rev. D 86, 084013 (2012).

[16] G. 't Hooft and M. J. G. Veltman, One loop divergencies in the theory of gravitation, Ann. Inst. Henri Poincaré, A 20, 69 (1974).

[17] L. D. Landau and E. M. Lifshitz, The Classical Theory of Fields (Pergamon Press, Oxford, 1975).

[18] R. Mertig, M. Bohm, and A. Denner, FeynCalc: Computer algebraic calculation of Feynman amplitudes, Comput. Phys. Commun. 64, 345 (1991).

[19] V. Shtabovenko, R. Mertig, and F. Orellana, New Developments in FeynCalc 9.0, Comput. Phys. Commun. 207, 432 (2016).

[20] R. L. Jaffe, The Casimir effect and the quantum vacuum, Phys. Rev. D 72, 021301 (2005).

[21] H. Nikolic, Proof that Casimir force does not originate from vacuum energy, Phys. Lett. B 761, 197 (2016). 